\title{
Laser-induced graphene for bioelectronics and soft actuators
}

\author{
Yadong $\mathrm{Xu}^{1}$, Qihui Fei ${ }^{1}$, Margaret Page ${ }^{2}$, Ganggang Zhao ${ }^{2}$, Yun Ling ${ }^{2}$, Dick Chen $^{3}$, and Zheng Yan ${ }^{1,2}(\bowtie)$ \\ ${ }^{1}$ Department of Biomedical, Biological \& Chemical Engineering, University of Missouri, Columbia, Missouri 65211, USA \\ ${ }^{2}$ Department of Mechanical \& Aerospace Engineering, University of Missouri, Columbia, Missouri 65211, USA \\ ${ }^{3}$ Rock Bridge High School, Columbia, Missouri 65203, USA
}

(C) Tsinghua University Press and Springer-Verlag GmbH Germany, part of Springer Nature 2021

Received: 13 November 2020 / Revised: 6 March 2021 / Accepted: 7 March 2021

\begin{abstract}
Laser-assisted process can enable facile, mask-free, large-area, inexpensive, customizable, and miniaturized patterning of laser-induced porous graphene (LIG) on versatile carbonaceous substrates (e.g., polymers, wood, food, textiles) in a programmed manner at ambient conditions. Together with high tailorability of its porosity, morphology, composition, and electrical conductivity, LIG can find wide applications in emerging bioelectronics (e.g., biophysical and biochemical sensing) and soft robots (e.g., soft actuators). In this review paper, we first introduce the methods to make LIG on various carbonaceous substrates and then discuss its electrical, mechanical, and antibacterial properties and biocompatibility that are critical for applications in bioelectronics and soft robots. Next, we overview the recent studies of LIG-based biophysical (e.g., strain, pressure, temperature, hydration, humidity, electrophysiological) sensors and biochemical (e.g., gases, electrolytes, metabolites, pathogens, nucleic acids, immunology) sensors. The applications of LIG in flexible energy generators and photodetectors are also introduced. In addition, LIG-enabled soft actuators that can respond to chemicals, electricity, and light stimulus are overviewed. Finally, we briefly discuss the future challenges and opportunities of LIG fabrications and applications.
\end{abstract}

\section{KEYWORDS}

laser-induced graphene, bioelectronics, biophysical sensors, biochemical sensors, soft actuators

\section{Introduction}

During the past decade, graphene, the most extensively studied two-dimensional (2D) material, has attracted broad attentions among the scientific community due to its outstanding and unique electrical, optical, mechanical, and thermal properties [1]. As a promising carbon nanomaterial with wide applications in catalysis, energy storage, electronics, optics, and many others, recent research has delved into exploring novel scalable fabrication methods of graphene [2, 3]. Currently, graphene can be produced with a variety of methods, such as mechanical exfoliation [4], chemical vapor deposition [5], liquid exfoliation [6], and flash joule heating [7]. However, these existing techniques usually suffer from tedious fabrication process, high energy consumption, and/or low productivity. In addition, the inability to achieve large-area, mask-free graphene patterning on versatile substrates, associated with these traditional approaches, inevitably constrains the wide adoption of graphene in emerging bioelectronics and soft robots.

Laser-assisted fabrication technique has emerged as a versatile and powerful alternative for the scalable fabrication of graphene on flexible substrates with customizable designs [8]. Direct laser reduction of graphene oxide (GO) was first reported by El-Kady et al. in 2012, where a standard LightScribe DVD optical drive was used to write graphene-based microelectronics [9]. In 2014, Tour et al. reported the fabrication of laser-induced porous graphene (LIG) on polyimide (PI) films using a commercial $\mathrm{CO}_{2}(10.6 \mu \mathrm{m})$ infrared (IR) laser system at ambient conditions [10]. This one-step process eliminates the need of GO precursors and significantly simplifies the fabrication process. Further studies revealed that visible laser and ultraviolet (UV) laser were also able to convert carbon precursors to LIG [11-13]. The transformation originates from photothermal effects for IR laser, photochemical effects for UV laser, and the combination of photothermal and photochemical effects for visible laser [14]. Specifically, the formation mechanism of LIG is dominated by an instantaneous pyrolysis process, where laser irradiation can give rise to a localized, high temperature $\left(>2,500^{\circ} \mathrm{C}\right)$, subsequently breaks chemical bonds in precursors, releases gaseous products, and rearranges carbon atoms to graphene [10]. The LIG morphology can be tailored by varying laser processing parameters, such as laser powers, speeds, and pulses per inch. The laser-assisted fabrication process is feasible to make various graphene-based flexible devices, largely because of its simple, scalable, mask-free fabrication process at ambient conditions, high flexibility with customizable pattern designs, and a plethora of potential carbon precursors as device substrates.

Since its discovery in 2014, LIG and its composites have been widely explored for a variety of applications (e.g., energy storage, catalysis, water treatment, flexible electronics, soft actuators). For example, the LIG-nanoparticle composite materials have been used as catalysts for many chemical reactions, such as oxygen reduction reaction, oxygen evolution reaction, hydrogen peroxide evolution. Some recent review papers have summarized the discovery and morphology control of LIG and its applications in energy storage (e.g., supercapacitors, fuel cells), catalysis, and microfluidics [15-19]. In this review 
paper, we mainly focus on the fabrication and properties of LIG and its applications in bioelectronics and soft robots, with the emphasis on the emerging research results reported in the past five years. In Section 2, we summarize fabrication approaches of LIG on various carbonaceous substrates and introduce its electrical, mechanical, and antibacterial properties and biocompatibility. In Section 3, we overview the recent studies of LIG-based bioelectronic devices, mainly including biophysical and biochemical sensors. Due to its high electrical conductivity and unique mechanical properties, LIG has been widely explored for biophysical sensors, such as strain sensors, pressure sensors, temperature sensors, hydration sensors, humidity sensors, and electrophysiological sensors. In addition, LIG exhibits outstanding electrochemical properties owing to its large surface areas and rich surface defects, showing promising applications in biochemical sensing. We provide a detailed summary of LIG-based biochemical sensors in terms of analytes (e.g., gas, electrolytes, metabolites, pathogens, nucleic acids, immunology) and detection mechanisms. Besides, we also introduce the applications of LIG in flexible energy generators and photodetectors. In Section 4, the recent research of LIG-based soft actuators and their applications in soft robots and three-dimensional (3D) assembly are presented. Finally, we briefly discuss the future challenges and opportunities of LIG fabrications and applications.

\section{Fabrications and properties of LIG}

Since the discovery of LIG in 2014, improved fabrication strategies and versatile precursor materials have been explored for making LIG and its derivatives with controlled morphologies and tailored properties. In this section, we mainly overview the LIG synthesis on various carbonaceous substrates and engineering of its porous structures, as well as introduce LIG's mechanical, electrical and antibacterial properties and biocompatibility that are all critical for bioelectronics and soft robots.

\subsection{LIG fabrications with various conditions and its electrical property}

LIG was first discovered accidently by Tour et al. in 2014 . They found that a commercial $\mathrm{CO}_{2}(10.6 \mu \mathrm{m})$ IR laser could be used to convert certain types of polymers, such as PI and polyetherimide (PEI), into porous graphene. The obtained LIG exhibits a high electrical conductivity. And, its sheet resistance can be optimized to $\sim 10 \Omega /$ sq. As shown in Fig. 1(a) [10], the instant laser irradiation results in localized, high temperatures,
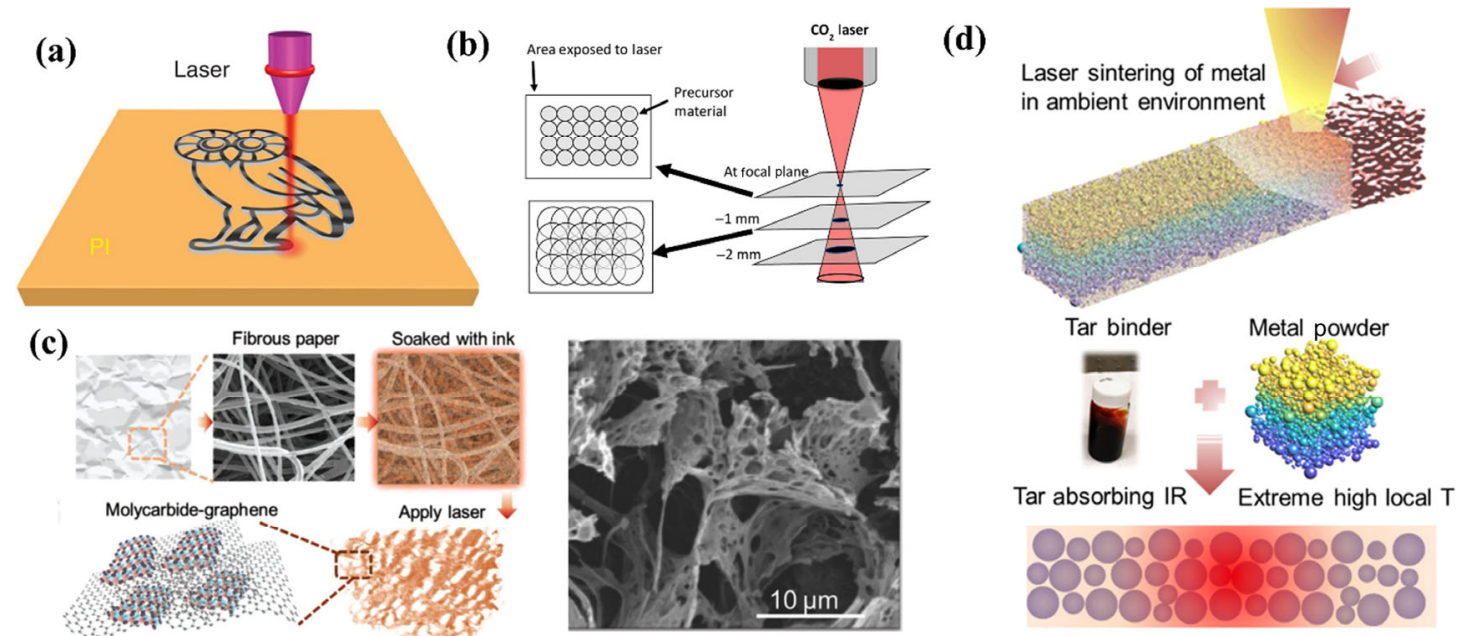

(c)
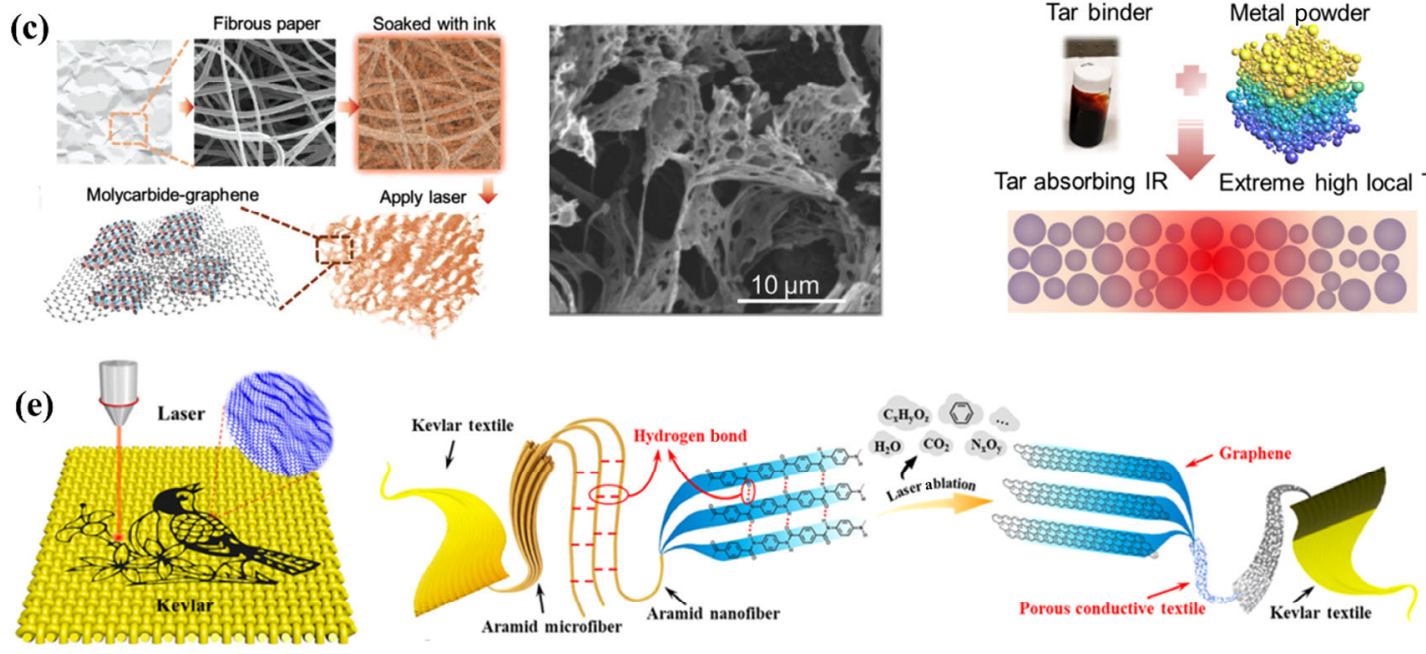

(f)
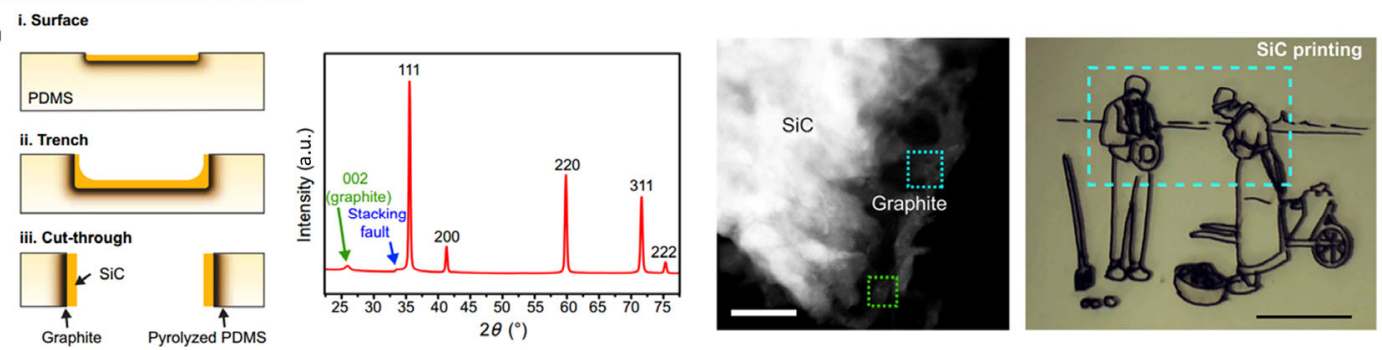

Figure 1 (a) Schematic illustration of the fabrication of LIG on PI. Reproduced with permission from Ref. [10], () Macmillan Publishers Limited 2014. (b) Diagram of the defocusing strategy to increase the laser spot size on the precursor substrate. Reproduced with permission from Ref. [31], $\odot$ American Chemical Society 2018. (c) Schematic illustration of the synthesis procedures and SEM image of the SiC-LIG composite made from gelatin/Mo ${ }^{5+}$ modified fibrous paper precursors. Reproduced with permission from Ref. [32], () WILEY-VCH Verlag GmbH \& Co. KGaA, Weinheim 2018. (d) Schematic illustration of laser-assisted fabrication of tar-mediated thin metal films. Reproduced with permission from Ref. [34], () American Chemical Society 2020. (e) Schematic illustration of the fabrication and formation process of LIG on Kevlar textiles. Reproduced with permission from Ref. [35], ๑ American Chemical Society 2020. (f) Illustration of direct laser scribing of the SiC-LIG composites on PDMS elastomer. Scale bars, $100 \mathrm{~nm}$ (left), and $1 \mathrm{~cm}$ (right). Reproduced with permission from Ref. [41], @ Nair, V. et al. 2020. 
which can break $\mathrm{C}-\mathrm{O}, \mathrm{C}=\mathrm{O}$, and $\mathrm{N}-\mathrm{C}$ bonds and rearrange carbon atoms to porous graphene structures (i.e., LIG). Recently, reactive force field (ReaxFF) simulations of the LIG formation on five different polymers further validate this theory, thus providing fundamental insights for LIG structural tailoring that can be leveraged for a rich variety of applications [20]. Some other types of polymers, which have similar molecular structures to PI, were also successfully utilized for LIG fabrications, including sulfonated poly(ether ether ketone) (SPEEK), poly (ether ether ketone) (PEEK), polysulfone (PSU), poly(ether sulfone) (PES), and polyphenylsulfone (PPSU) [21-24]. The obtained LIG has intrinsic sulfur doping inherited from the precursor materials, which exhibits the enhanced electrocatalytic property. Besides, Zhang et al. reported the fabrication of LIG on phenolic resin using a $405 \mathrm{~nm}$ semiconductor laser under ambient conditions [25]. Cao et al. synthesized LIG on a polybenzoxazine resin poly ( $\mathrm{Ph}-\mathrm{ddm}$ ) substrate using a $\mathrm{CO}_{2}$ IR laser [26]. The fabricated LIG exhibits outstanding resistance and durability to extreme physical and chemical conditions. Recently, extending precursor materials to an inexpensive and renewable alternative, Tour et al. synthesized LIG on pine wood under the protection of Ar or $\mathrm{H}_{2}$ gases, which can avoid ablation of precursor materials in air [27]. In the study, the crosslinked lignocellulose structures inside wood were demonstrated to be more preferable in the formation of LIG. Some other examples regarding lignin-based LIG synthesis were also reported recently [28-30]. Furthermore, as illustrated in Fig. 1(b), Tour et al. has improved the laser scribing process, where a defocused laser or multiple lasing were used to first convert precursors to amorphous carbons, followed by the transformation of amorphous carbons to graphene [31]. This technique eliminates the need of protection gases described above and can enable the generation of LIG on a rich variety of carbonaceous substrates (e.g., food, cloth, paper, cardboard, natural coal) at atmospheric conditions.

Recently, Zang and her coworkers have developed a direct IR laser scribing technique for the fabrication of conductive molybdenum carbide $\left(\mathrm{Mo}_{3} \mathrm{C}_{2}\right)$ and $\mathrm{LIG}$ composites on paper substrates, as demonstrated in Fig. 1(c) [32]. The fabrication process is assisted with the gelatin-mediated inks containing molybdenum (Mo) ions, which are spray-coated onto paper substrates, followed by laser-induced generation of $\mathrm{Mo}_{3} \mathrm{C}_{2}$ and LIG composites. The effective IR absorption by Mo-gelatin composites can give rise to localized, high temperatures, thus leading to the liberation of gaseous compounds and recrystallization of carbon atoms. The simultaneous carbonization of Mo ions and gelatin results in the formation of $\mathrm{Mo}_{3} \mathrm{C}_{2}$ and LIG composites. The fabricated composites exhibit high electrical conductivity ( $30 \Omega / \mathrm{sq})$ and highly porous structures as shown in Fig. 1(c). Besides, ultrathin transition metal carbides including molybdenum carbide, tungsten carbide, and cobalt carbide were also fabricated by the same group using similar procedures [33]. Very recently, tar was employed as both IR absorber and antioxidant binder for laser-assisted fabrication of thin metal films and metal carbides as shown in Fig. 1(d) [34]. Here, the carbon solid solubilities and formation energies of metal carbides are two critical factors that determine the formation of carbides. In this study, aluminum (Al), copper $(\mathrm{Cu})$ and nickel $(\mathrm{Ni})$ were sintered to generate metal thin films, whereas Mo and tungsten (W) were converted to carbides with exceptional electrical conductivities $(\sim 0.1 \Omega / \mathrm{sq})$.

Direct patterning of electronic components on flexible substrates is highly preferred for bioelectronics and soft robots. Laser-assisted process provides a facile, mask-free, highthroughput technique for the fabrication of conductive LIG patterns on various flexible substrates. As illustrated in Fig. 1(e),
Wang et al. adopted a laser scribing process to write LIG directly on Kevlar textile in air and explored its application as an intelligent protective clothing [35]. The outstanding selfextinguishing capability of Kevlar textiles, which originates from the covalent bonds between the aromatic nucleus and imide, as well as hydrogen bonds between the carbonyl and $\mathrm{N}-\mathrm{H}$ groups, can result in a reduced photon absorption with a $\mathrm{CO}_{2}$ IR laser to avoid laser ablation. Therefore, the photothermal effect induced by direct laser irradiation assists the formation of LIG. In addition, it is known that elastomers are well suitable supporting substrates for soft bioelectronics and soft actuators due to their high stretchability, biocompatibility, and tissue-like compliance. However, elastomers are not ideal materials for LIG synthesis, mainly owing to their low melting points (e.g., poly(styrene-ethylene-butylene-styrene)) or low carbon contents (e.g., silicone elastomers). Thus, LIG is usually transferred from mother precursor substrates (e.g., PI) onto elastomers for soft device fabrication [36-38]. It is worth noting that some recent research has explored the direct fabrication of other electronic materials, such as silicon carbide ( $\mathrm{SiC}$ ) on silicone elastomers. For example, SiC-enabled strain sensors were made via direct lasing on Ecoflex elastomers using a $532 \mathrm{~nm}$ continuous laser beam [39]. X-ray diffraction (XRD) analysis reveals that laser-scribed siloxane is converted to $3 \mathrm{C}-\mathrm{SiC}$ and $4 \mathrm{H}-\mathrm{SiC}$. The fabricated functional materials demonstrate relatively low electrical conductivities. Most recently, laser-assisted $\mathrm{SiC}$ fabrications on polydimethylsiloxane (PDMS) were also demonstrated [40, 41]. As illustrated in Fig. 1(f), nitrogen-doped cubic silicon carbide $(3 \mathrm{C}-\mathrm{SiC})$ is produced on the PDMS surface using a $\mathrm{CO}_{2}$ IR laser under a nitrogenrich atmosphere [41]. A thin layer of spongy graphite is simultaneously formed to bridge the PDMS matrix and $\mathrm{SiC}$, which is confirmed by scanning transmission electron microscopy (STEM) images and XRD spectrum (Fig. 1(f)). The obtained materials exhibit very good pseudocapacitive electrochemical and photoelectrochemical properties. Their applications in in vitro electrocardiograms (ECG) recording and stimulations of primary human aortic smooth muscle cells were demonstrated. Table 1 provides a summary of LIG and its composites prepared with different conditions, as well as their electrical properties.

\subsection{Porous structures engineering of LIG and its supporting substrates}

LIG typically demonstrates multiscale porous structures owing to instantaneous liberation of gaseous compounds during the laser irradiation process, thereby providing a high surface area of around $340 \mathrm{~m}^{2} / \mathrm{g}$ [10]. However, the high temperature associated with the laser-assisted fabrication inevitably distorts the substrate polymer structure, thus compromising their performance in the applications where micro- or nano-sized porous features are preferred. This is particularly essential for the applications ranging from membrane filtration, sensing, catalysis, and energy storage to breathable bioelectronics $[42,43]$. Recently, our research group has adopted an indirect approach to fabricate LIG-based porous skin-interfaced bioelectronics with high breathability [37]. In this research, LIG fabricated on the PI substrate is first laminated onto partially cured, sugar-templated PDMS, followed by the solidification and sugar dissolution to achieve the transfer of porous LIG onto porous PDMS substrates. The porous structures of both LIG and PDMS are well preserved after the transfer. And, the obtained porous skin-interfaced bioelectronic devices are highly breathable, soft, and stretchable. Besides, Tan et al. recently reported an in situ, instantaneous generation 
Table 1 LIG and its composites prepared with different conditions and their electrical properties

\begin{tabular}{|c|c|c|c|c|}
\hline Precursors & Laser sources & Lasing conditions & Electrical properties & Ref. \\
\hline Phenolic resin & Visible & Air & $\sim 44 \Omega / \mathrm{sq}$ & [25] \\
\hline PI, PEI, SPEEK, PEEK, PSU, PES, PPSU & IR & Air & $\sim 10-25 \Omega / \mathrm{sq}$ & {$[10,21-24]$} \\
\hline Poly $(\mathrm{Ph}-\mathrm{ddm})$ & IR & Air & $\sim 35 \Omega / \mathrm{sq}$ & [26] \\
\hline Wood & IR & Ar or $\mathrm{H}_{2}$ & $\sim 10 \Omega / \mathrm{sq}$ & [27] \\
\hline Lignin-based materials & IR & Air & $\sim 3.8-400 \Omega / \mathrm{sq}$ & [28-30] \\
\hline Food, cloth, paper, cardboard, natural coal & IR & Air & $\sim 5 \Omega / \mathrm{sq}$ & [31] \\
\hline Mo ion doped gelatin & IR & Air & $\sim 30 \Omega / s q$ & [32] \\
\hline Metallo-hydrogel & IR & Air & $\sim 300 \mathrm{~S} / \mathrm{cm}$ & [33] \\
\hline Kevlar textile & IR & Air & $\sim 10.6 \Omega / \mathrm{sq}$ & {$[35]$} \\
\hline Silicone elastomers & Visible or IR & Air or $\mathrm{N}_{2}$ & Not available & {$[39,41]$} \\
\hline PI & UV & Air & $\sim 24 \Omega / \mathrm{cm}$ & {$[11]$} \\
\hline PI & Visible & Air & $\sim 108 \Omega /$ sq & [12] \\
\hline
\end{tabular}

of 3D hierarchically porous carbon structures using block copolymers with meso- and macroscopic features as precursors assisted with transient $\mathrm{CO}_{2}$ laser heating [44]. A schematic of the fabrication process is provided in Fig. 2(a). The structuredirecting block copolymer is first mixed with resols to form an organic hybrid thin film. And, subsequent laser treatment generates localized heating, which leads to the simultaneous thermopolymerization of resols and decomposition of block copolymers. This process induces the formation of well-defined hierarchically porous structures with a thickness of $\sim 200 \mathrm{~nm}$ and pore sizes spanning from tens to hundreds of nanometers (Fig. 2(b)) [44]. Finally, nanoporous carbon films with electrical conductivity up to $270-750 \mathrm{~S} / \mathrm{cm}$ are obtained through further furnace heating under nitrogen atmosphere. During the fabrication process, the pristine porous resol structures are well preserved without noticeable collapse. Most recently, a method associated with sequential infiltration synthesis (SIS) of alumina has been developed to preserve the porous structures of polymer precursor substrates during the LIG fabrication [45]. Specifically, SIS allows the penetration and in situ formation of alumina within the porous structures of the polymer substrates by using alternating pulses of trimethylaluminum (TMA) and water as shown in Fig. 2(c). With this technique, porous PES microfiltration membranes are adopted as model substrates, on which conductive and electrochemically stable LIG is formed after laser irradiation. And, the porosity of the underlying PES substrate is well maintained (Fig. 2(d)). The working mechanism lies in the enhanced thermal resilience toward laser pyrolysis, which can help maintain the porous features and high permeability of PES precursor substrates. Also, LIG obtained on porous PES with SIS treatment exhibits a high electrical conductivity $(\sim 37.7 \Omega / \mathrm{sq})$, in contrast to its counterpart $(>1,000 \Omega /$ sq) fabricated on porous PES without SIS treatment.

\subsection{Mechanical properties}

Ideally, LIG-based biointegrated electronic devices need to possess high mechanical compliance (i.e., low elastic modulus) and high stretchability to match soft, curvilinear, dynamic biological tissues. For example, natural deformation of biological tissues can induce artifacts of bioelectronics and inevitably compromise the fidelity and reliability of acquired signals. Currently, high-quality LIG is generally fabricated on flexible
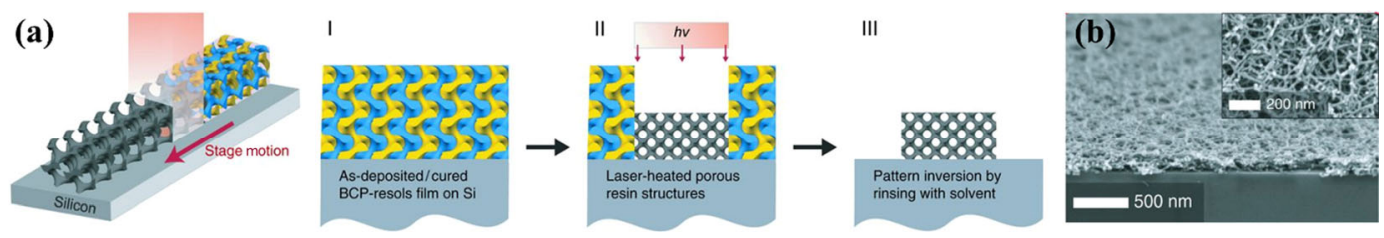

(c)
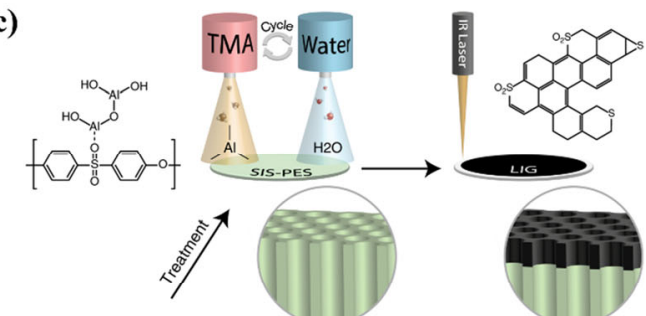

(d)
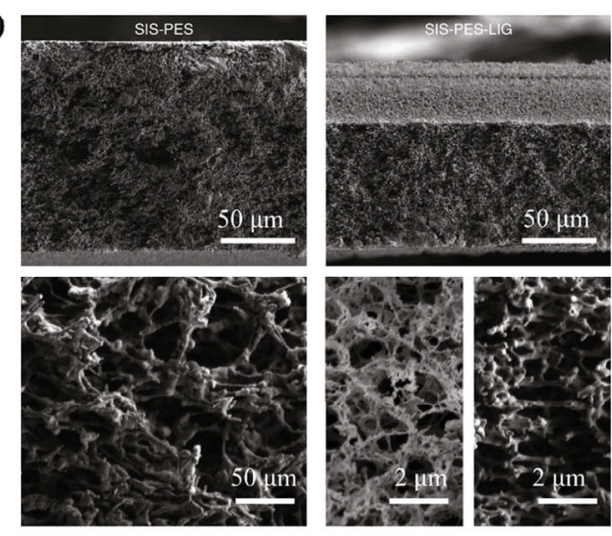

Figure 2 (a) Schematic illustration of transient laser heating of block copolymer-directed resols. (b) Cross-section SEM images of the porous structures obtained from process (a). Reproduced with permission from Ref. [44], () American Association for the Advancement of Science 2015. (c) Schematic illustration of laser-induced graphitization of PES membranes with (top) and without (bottom) SIS of alumina. (d) Cross-section SEM images of the SIS treated precursors (left) and obtained LIG-PES composites (right), indicating well preserved porous structures of PES after an IR laser irradiation process. Reproduced with permission from Ref. [45], ๑ Bergsman, D. S. et al. 2020 
thermoplastic substrates (e.g., PI, PEI, PES), which usually exhibit excellent mechanical robustness and outstanding durability but suffer from high stiffness and poor stretchability. Figure 3(a) demonstrates the resistance variations of LIG fabricated on PI as a function of bending radius and cyclic bending cycles [46]. The compression or stretching induced by inward or outward bending results in the increase or decrease of the LIG's electrical conductivity. Cao et al. reported the LIG fabrication on poly (Ph-ddm), which demonstrates outstanding mechanical integrity as evidenced by repeated adhesion tests (Fig. 3(b)) [26]. This is important for biointegrated electronic devices since potential leakages caused by poor mechanical integrity may pose severe safety risks. Also, several studies have shown the good performance retention of LIG-based devices under mechanical deformations [47-49]. Despite the recent advances in LIG-enabled bioelectronics [50, 51], the inability of LIG made on thermoplastic substrates to stretch poses significant challenges in realizing high-fidelity biointegrated electronic devices. To overcome this handicap, by taking the advantage of screen printing, a composite ink, consisting of poly(3,4-ethylenedioxythiophene):polystyrene sulfonate (PEDOT:PSS), LIG powders, and polyurethane (PU), has been developed (Fig. 3(c)) [52]. The screen-printed device can be laminated on the human skin and maintains mechanical integrity when it subjects to twisting, stretching, and indentation due to the low elastic modulus and high stretchability afforded by PU matrixes. And, high capacitance retention under large strain (100\%) and cycling stretching (200 cycles) indicates robustness of the screen-printed devices (Fig. 3(d)) [52]. In addition, transferring LIG from PI mother substrates to stretchable elastomer substrates is one simple yet efficient strategy to fabricate soft bioelectronics [28, 36-38, 53]. Recently, our research group further improved the stretchability of LIG transfer-printed on PDMS substrates by adopting a kirigami design [37]. As shown in Fig. 3(e), negligible resistance variations of obtained device are observed under up to $800 \%$ uniaxial strains. In addition, with a mechanically guided deterministic assembly approach that typically is employed for fabrications of 3D metal, polymer and silicon structures, our research group has demonstrated the assembly of more than 10 different LIG-based 3D architectures that utilize kirigamiand origami-based designs [54]. The process starts with the fabrication of 2D LIG-PI precursors with predesigned patterns, followed by a mechanically guided assembly process induced by the strain release of prestretched elastomer substrates. Notably, the resulting 3D helical structures of LIG exhibit outstanding electromechanical performances under bending and stretching testing (Fig. 3(f)). The enabled LIG-based 3D helical supercapacitors show unnoticeable areal capacitance changes under a biaxial strain of $100 \%$ and only decrease by 9\% after 1,000 times of cyclic stretching tests.

\subsection{Antibacterial property and biocompatibility}

Early studies in the LIG's application of water treatment provided insights into its biofilm resistance and antibacterial property [55]. One recent study has demonstrated the LIG's intrinsic antibacterial capability after $8 \mathrm{~h}$ incubation of Escherichia coli (E. coli) [56]. As illustrated in Figs. 4(a) and 4(b), the scanning electron microscopy (SEM) images and epifluorescence microscopy images (green for live bacteria and red for dead bacteria) showcase the LIG's intrinsic bactericidal capability. Statistical studies reveal that the viability of E. coli. significantly drops on LIG, which gives an antibacterial efficacy of $81.57 \%$. Notably, the surface properties of LIG can be tuned by adjusting
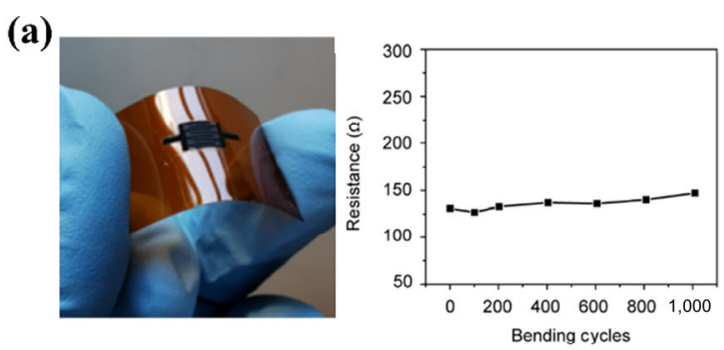

(c)

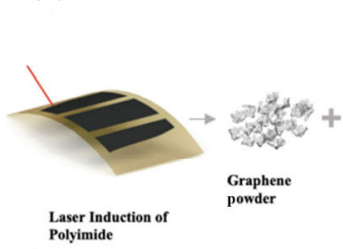

(e)
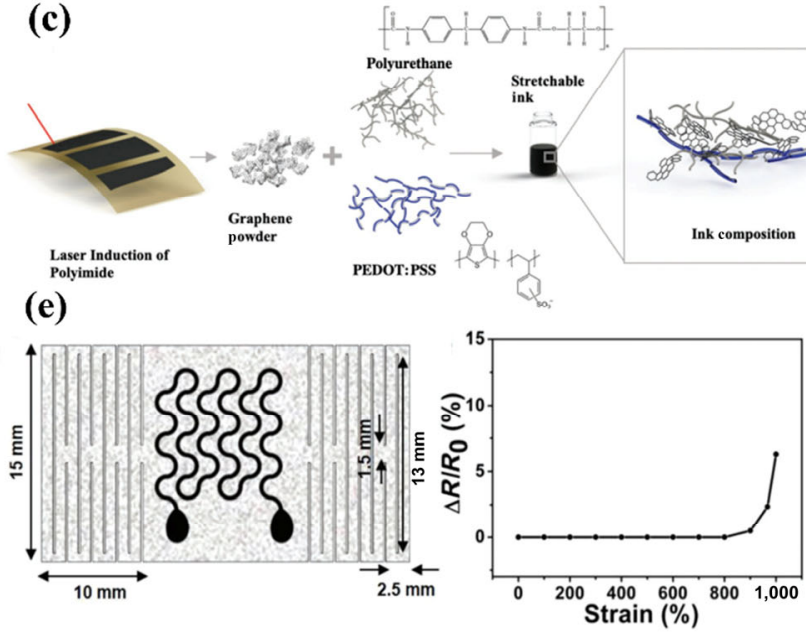

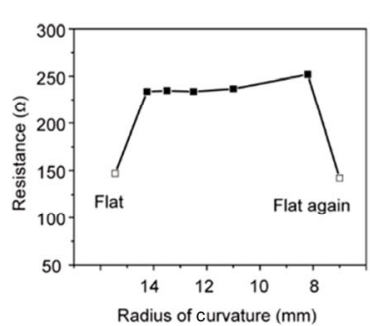

(d)
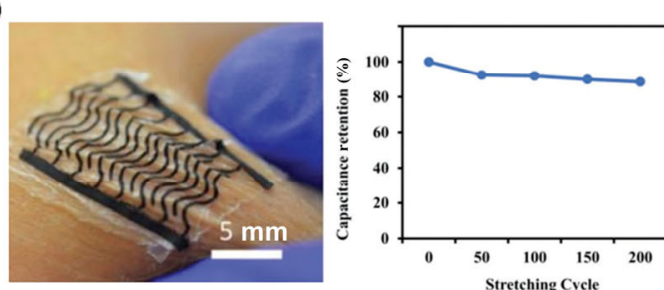

(f)
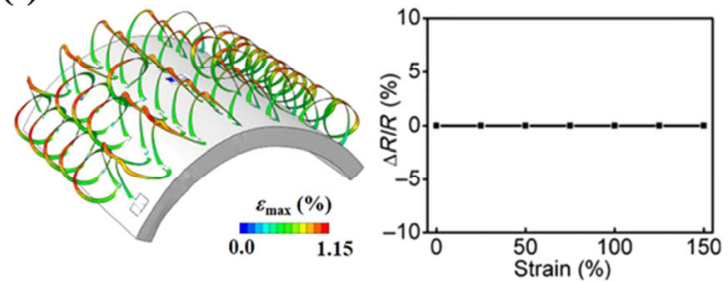

Figure 3 (a) A photo of LIG on PI and its corresponding electromechanical properties. Reproduced with permission from Ref. [46], (C) American Chemical Society 2020. (b) Electrical variations of LIG fabricated on poly (Ph-ddm) substrates under multiple scotch tape adhesion tests. Reproduced with permission from Ref. [26], (c) Elsevier Ltd. 2020. (c) Schematic illustration of making LIG-based inks for screen printing. (d) The conformal contact between the screen-printed device and skin, and capacitance retentions under cyclic stretching tests. Reproduced with permission from Ref. [52], (C) WILEY-VCH Verlag GmbH \& Co. KGaA, Weinheim 2019. (e) LIG patterns transfer-printed on silicone elastomers with kirigami cuts, indicating high stretchability. Reproduced with permission from Ref. [37], (c) WILEY-VCH Verlag GmbH \& Co. KGaA, Weinheim 2018. (f) Finite element analysis of 3D helical coils of LIG and its resistance change under stretching. Reproduced with permission from Ref. [54], (c) American Chemical Society 2018. 
(a)

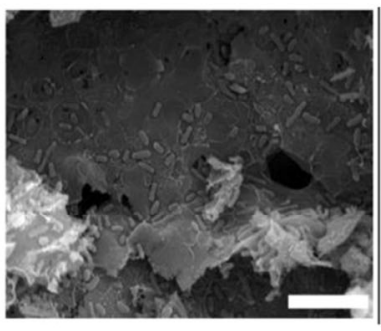

(c)

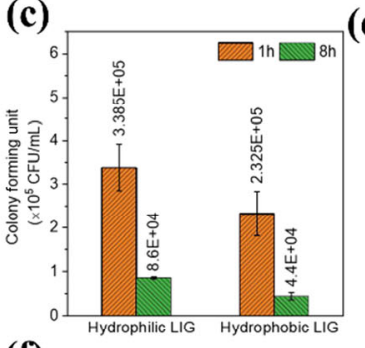

(f)

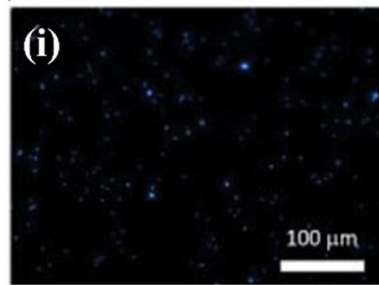

(d)
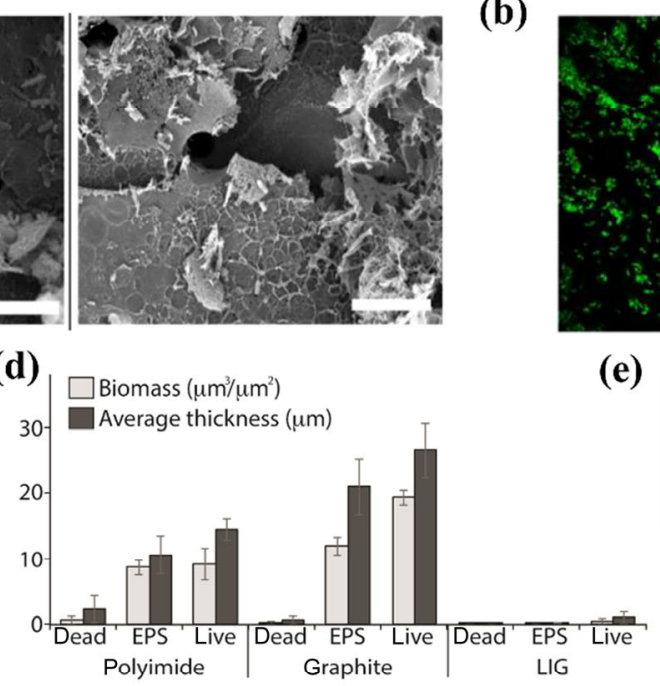

(e) (b)
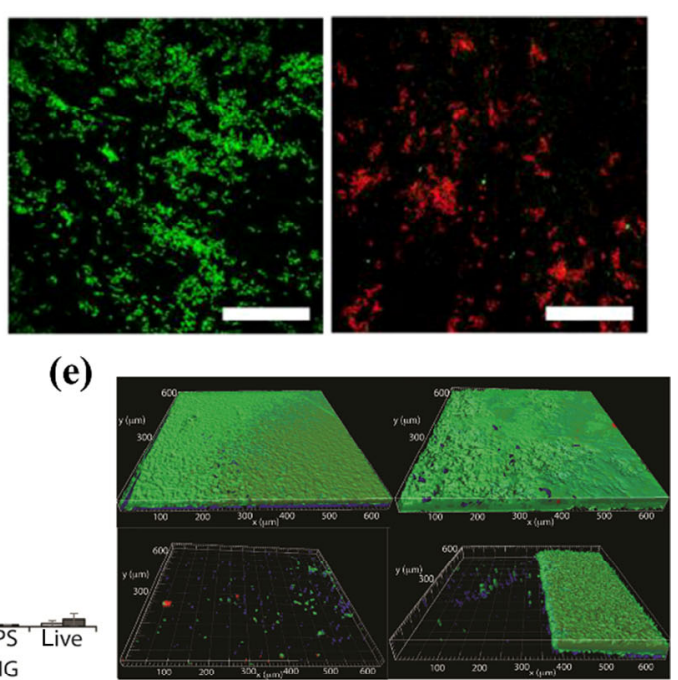
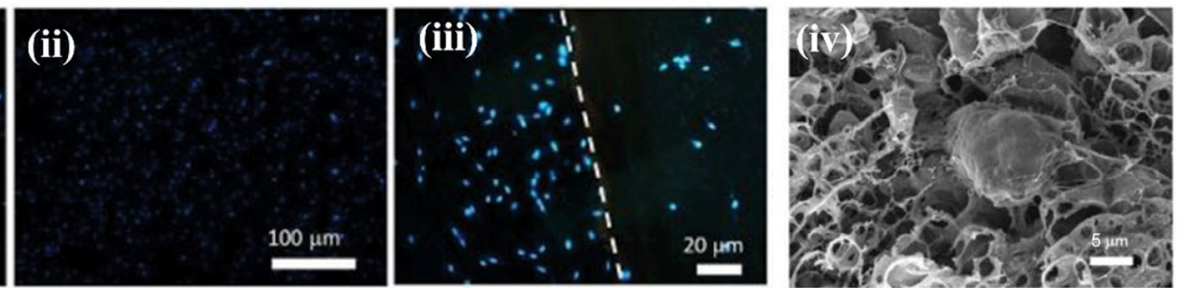

Figure 4 SEM images (a) and laser scanning confocal microscopy images (b) of LIG after 1 and $8 \mathrm{~h} \mathrm{E}$. Coli incubation. (c) Statistic analysis of the CFU assay on hydrophilic and hydrophobic LIG. Reproduced with permission from Ref. [56], ( ) American Chemical Society 2020. (d) Biofilm growth in terms of biomass and average thickness on the surfaces of PI, graphite, and LIG with P. aeruginosa. (e) IMARIS software images for the PI, graphite, LIG, and interface between LIG (left) and PI film (right). Green represents live bacteria, red represents dead bacteria in (b) and (e), and blue represents EPS in (e). Reproduced with permission from Ref. [55], () American Chemical Society 2017. (f) Fluorescence microscope images of MDA-MB-231 cells on LIG after $24 \mathrm{~h}$ (i) and $48 \mathrm{~h}$ (ii) of incubation, the interface between the LIG and PI film (iii), and a SEM image of one single cell on LIG (iv). Reproduced with permission from Ref. [60], ( WILEY-VCH Verlag GmbH \& Co. KGaA, Weinheim 2020.

laser scribing parameters. Figure 4(c) shows that hydrophobic LIG exhibits $7 \%$ bactericidal improvement over hydrophilic LIG [56]. Besides, Tour et al. have recently studied the biofilm formation on PI, graphite, and LIG with Pseudomonas aeruginosa (P. aeruginosa) as the model example [55]. In contrast to PI and graphite, LIG can effectively resist the biofilm formation in terms of life and dead cells adhesion and extracellular polymeric substance (EPS) accumulation, as evidenced in Figs. 4(d) and 4(e). This antifouling capability is ascribed to the LIG's less negative zeta potential and hydrophilic surface. Some strategies have been used to further amplify the LIG's antimicrobial capability. For example, applying external voltages can amplify the LIG's antimicrobial performance because of the synergic electrical and chemical effects (e.g., in situ formation of hydrogen peroxide) $[22,55]$. In addition, by leveraging its photothermal property, LIG has been integrated on commercial face masks to provide the self-cleaning capability and antibacterial property in response to the 2019 coronavirus (COVID-19) outbreak $[56,57]$.

A major concern associated with the applications of LIGbased biointegrated electronic devices is the long-term biocompatibility and toxicity. Despite the recent in vivo demonstration of implanted LIG electrodes [58], its toxicological studies on biological applications are still rarely reported. Until very recently, a toxicity assessment of LIG was carried out on zebrafish (Danio rerio) due to its high homology with humans [59]. The effects of LIG administration with various concentrations $(5-100 \mu \mathrm{g} / \mathrm{mL})$ were investigated by monitoring the growth, hatching, and development of zebrafish embryos. The results demonstrated the negligible effects of LIG in zebrafish and its larval behavior, indicating good biocompatibility.
Besides, Wang et al. evaluated the biocompatibility of LIG using human breast cancer cells (MDA-MB-231) through 24 and $48 \mathrm{~h}$ incubation [60]. Figure 4(f) provides fluorescence microscope images of MDA-MB-231 cells, showing their continuous proliferation on LIG over $48 \mathrm{~h}$ incubation. Moreover, the cells exhibited preferential adhesion on LIG over PI, as illustrated in the SEM image. Another study also validates the biocompatibility of LIG by using a strain II of the epithelial cell line Madin-Darby Canine Kidney (MDCK-II) as the model example [61].

\section{LIG-based bioelectronic devices}

Soft biointegrated electronic devices are becoming increasingly important for next-generation health monitoring and management as they can be intimately laminated on human organs (e.g., skin) for minimally invasive, continuous, real-time, and long-lasting data acquisition, thus dynamically picturing the body's states [62, 63]. Laser-assisted, large-area, and mask-free patterning of graphene on versatile substrates provides a facile, cost-effective approach of making graphene-based bioelectronics compared to tedious and complicated lithography-based fabrications. At present, various types of LIG-based bioelectronic devices have been successfully developed to monitor of a variety of biophysical and biochemical signals [14, 16-19]. In this section, we summarize the recent progress of developing bioelectronic devices based on both pristine and functionalized LIG. The applications of LIG in flexible energy generators and photodetectors are also introduced.

\subsection{LIG-based biophysical sensors}

A broad spectrum of LIG-based biophysical sensors, such as 
strain, pressure, temperature, hydration, and electrophysiological sensors, have been developed to monitor vital physiological signals from bodies by taking advantage of its high electrical conductivity and high deformation-induced conductivity change (Table 2).

\subsubsection{Strain and pressure sensors}

LIG-based strain sensor was first reported by transferring pristine LIG from PI precursor substrates to PDMS elastomers [36]. The obtained strain sensor with the gauge factor (GF) up to 20,000 and stretchability of $100 \%$ was employed for detecting finger motions. In addition, UV laser irradiation on PI substrates can produce LIG with a high spatial resolution $(\sim 50 \mu \mathrm{m})$ (Fig. 5(a)) [11]. As shown in Figs. 5(b) and 5(c), by optimizing the substrate thickness and LIG morphology, the obtained LIG strain sensor exhibits a hysteresis-free linear response with a GF of $\sim 20$ and is used for radial and carotid pulse waveforms monitoring. By leveraging strain-induced resistance variations, many other LIG-based strain sensors have been developed. For example, the LIG decorated with $\mathrm{MoS}_{2}$ [64] or embedded in soft medical-grade PU [65], and SiC-LIG composite $[39,40]$ were explored for strain sensing. Typical applications involve pulse waveform monitoring [40, 64, 66], motion detection [64, 67-69], smart gear [24], respiration detection $[50,65,70]$, and underwater animal speed monitoring [71]. Besides, LIG can also be employed for thermoacoustic sound generation and detection. For instance, Tao et al. have recently developed an intelligent LIG artificial throat using a $450 \mathrm{~nm}$ laser source, which can simultaneously generate and detect sound within a single device (Fig. 5(d)) [51]. The working mechanism of the LIG-based sound detector lies in small resistance variations in response to minute air vibrations when a bias voltage is applied, whereas the LIG-based sound generation relies on the periodic joule heat generated by alternating current (AC) voltages which cause air expansion and therefore sound wave generation. As shown in Figs. 5(e) and 5(f), the LIG-enabled artificial throat can distinguish various throat vibrations that are caused by throat movements, and hum tones and intensities [51]. The artificial throat indicates exceptional reproducibility and robustness, which allows possible realization of sound recognition and identity authentication by integrating with machine learning algorithms. By taking

Table 2 LIG-based biophysical sensors

\begin{tabular}{|c|c|c|c|c|}
\hline Biophysical signals & Possible modifications & $\begin{array}{l}\text { Measurement } \\
\text { methods }\end{array}$ & Potential applications & Ref. \\
\hline Strain/pressure & $\begin{array}{l}\mathrm{MoS}_{2}, \mathrm{SiC}, \text { AgNPs, PS } \\
\text { microspheres }\end{array}$ & Resistive change & $\begin{array}{l}\text { Monitoring of pulse waveform, motion, respiration, } \\
\text { and blood pressure, and artificial throat }\end{array}$ & $\begin{array}{l}{[11,36,39,51} \\
64,66,72,73]\end{array}$ \\
\hline Temperature & None & Resistive change & Body temperature measurement & {$[37,75,76]$} \\
\hline Hydration & None & Impedance change & Monitoring of skin hydration levels & {$[37]$} \\
\hline Humidity & None & $\begin{array}{l}\text { Resistive, impedance } \\
\text { change }\end{array}$ & $\begin{array}{c}\text { Monitoring of human breath and plant } \\
\text { transpiration }\end{array}$ & {$[12,30,80]$} \\
\hline $\begin{array}{l}\text { Electrophysiological } \\
\text { signals }\end{array}$ & AgNWs & $\begin{array}{l}\text { Biopotential } \\
\text { measurement }\end{array}$ & $\begin{array}{l}\text { ECG, EMG and EEG recording, and neural sensing } \\
\text { (SEPs) }\end{array}$ & {$[35,37,83]$} \\
\hline
\end{tabular}

(a)

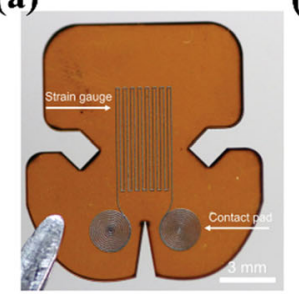

(d)

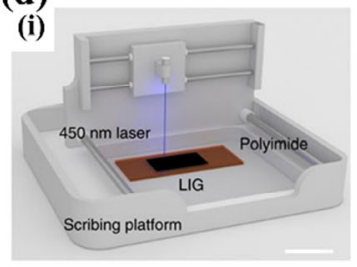

(e)

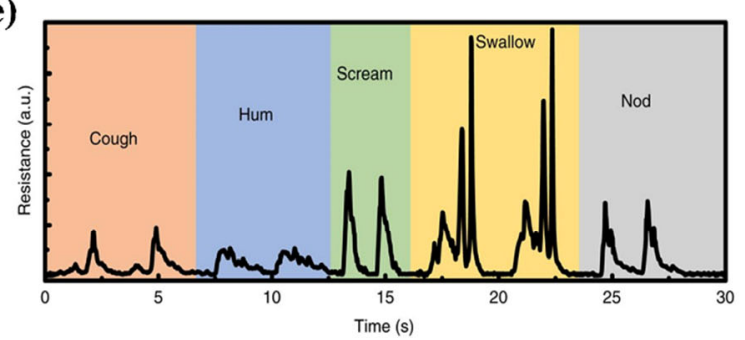

(b)

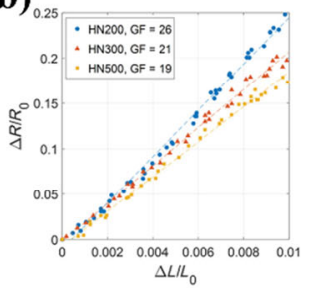

(ii)

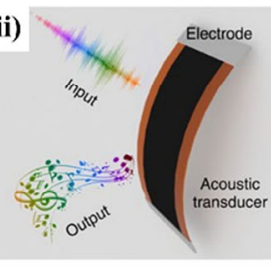

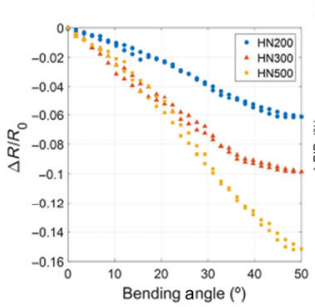

(c)
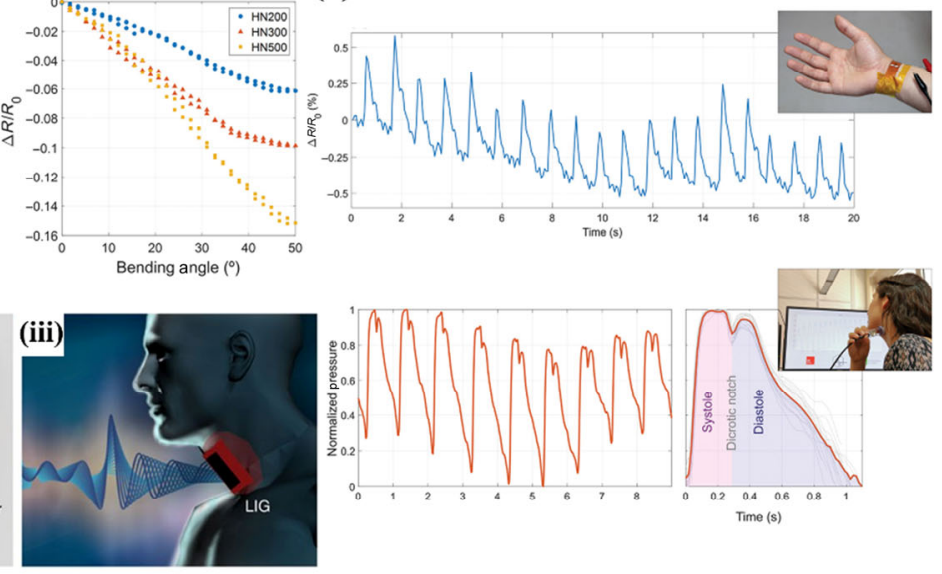

(f)
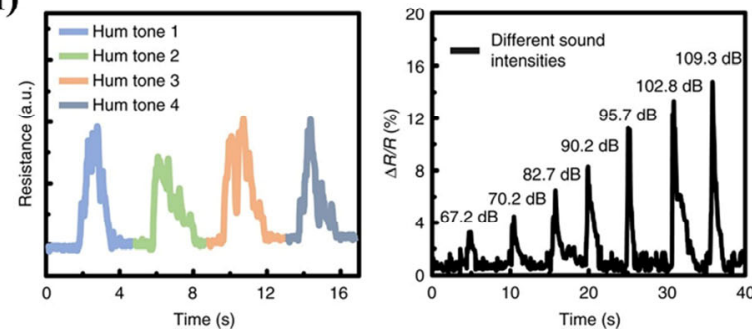

Figure 5 (a) A photograph of a LIG-based strain gauge fabricated on a Kapton sheet. (b) Piezoresistive characterizations of the strain gauge under stretching and bending tests. (c) The applications of LIG-based strain gauge for arterial and carotid pulse detections. Reproduced with permission from Ref. [11], (c) WILEY-VCH Verlag GmbH \& Co. KGaA, Weinheim 2018. (d) Schematic illustration of the fabrication process of the LIG-based artificial throats and the characterizations. The enabled device can response toward different kinds of throat vibrations (e) and hum tones and intensities (f). Reproduced with permission from Ref. [51], (c) Tao, L.-Q. et al. 2017. 
advantage of the LIG sound transducer and speaker, Chen et al. reported a dual-functional health monitoring electronic skin system with self-alarm capabilities [66]. The device can perform physiological signal recording such as wrist pulse waveforms and respiration rates and simultaneously provide sound alarms when predetermined abnormal situations occur.

Rahimi et al. fabricated porous LIG and silver nanoparticle (AgNPs) composites by thermal reduction of AgNPs that were selectively deposited onto LIG [72]. The composite materials exhibit excellent electrical conductivity of $0.02 \Omega /$ sq and have been used for flexible and wireless inductor-capacitor (LC) passive pressure sensors. Inspired by the bean pod structure, polystyrene microspheres with different diameters are sandwiched between two LIG layers to prepare resistive pressure sensors, which show significantly improved sensitivity, wide sensing ranges, and good stability as compared to pristine LIG-based devices owing to enhanced spacing effects [73]. In addition, together with the simultaneous measurement of other physiological signals, LIG-based pressure sensors can be used for blood pressure measurement. For instance, our research group has developed an LIG-based on-skin healthcare diagnostic device that can provide concurrent monitoring of ECG and arterial pulse waveforms [74]. Figure 6(b) provides schematic illustrations of working mechanisms, where LIG-based piezoresistive pressure sensors are used for pulse waveforms recording and electrophysiological sensors are adopted for simultaneous ECG measurement. The pressure sensor consists of LIG sensing materials and two supporting elastomer layers with one of which printed with interdigitated conductive electrodes (Fig. 6(a)). The beat-to-beat blood pressures can be determined from the pulse transit times (PTT) that have quantitative correlations with both diastolic and systolic blood pressures (Figs. 6(c) and 6(d)). The determined results agree well with the ones measured from a commercial sphygmomanometer.

\subsubsection{Temperature sensors}

LIG has unique features that can be utilized for thermal monitoring. For example, electrical conductivity changes caused by the electron-phonon scattering and thermal velocity of electrons in response to temperature variations [75] can be adopted for developing LIG-based temperature sensors. Pristine LIG made on PI under vector modes exhibits a sensitivity of $-0.06 \%{ }^{\circ} \mathrm{C}^{-1}$ (a)

(c)
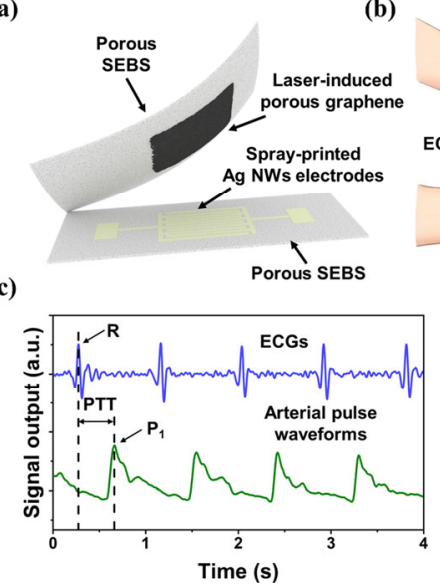

(b)

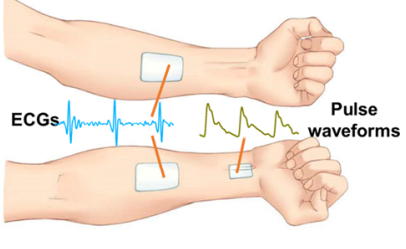

(d)

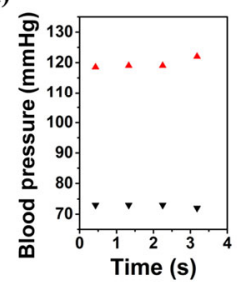

Figure 6 (a) A schematic diagram of the assembled LIG-based piezoresistive pressure sensor. (b) Schematic illustration of concurrent recording of ECGs and arterial pulse waveforms for blood pressure monitoring. (c) Recorded concurrent ECG signals and arterial pulse waveforms with the PTT indicated. (d) The determined beat-to-beat systolic (red) and diastolic (black) blood pressures based on the PTT method. Reproduced with permission from Ref. [74], (C) Xu, Y. D. et al. 2020. and a detection limit of $0.051{ }^{\circ} \mathrm{C}$ and thus can be used for on-skin temperature monitoring [50]. The results agree well with the work of Bobinger et al. [76], which gives a negative coefficient of $-0.46 / \mathrm{m}^{\circ} \mathrm{C}$. By transferring LIG onto porous PDMS substrates, however, the obtained temperature sensor exhibits a significantly high yet positive temperature coefficient of $0.04{ }^{\circ} \mathrm{C}^{-1}$ [37]. The difference can probably be attributed to the porous elastomer supporting substrate, which expands upon thermal heating. In addition, LIG has also been used as jouleheating elements due to its excellent conductivity ( $\sim 10 \Omega / \mathrm{sq})$. The enabled joule heaters can operate well at temperatures up to $400{ }^{\circ} \mathrm{C}[37,76]$.

\subsubsection{Hydration and humidity sensors}

Skin hydration is indicative of the dynamic physiological status and is therefore useful in many applications, such as evaluations of cosmetics, mental stress and some skin diseases (e.g., eczema). Our research group has developed an LIG-based on-skin hydration sensor, which can monitor skin impedance variations at various skin hydration levels [37]. Higher hydration levels typically lead to lower skin impedances [77]. Our studies find that the impedance change can be amplified at low measurement frequencies [37]. The obtained results demonstrate a good agreement between the LIG-based hydration sensor and a commercial corneometer. Humidity sensors have also been reported recently by using interdigital porous LIG as highly conductive electrodes and lignin [30] or GO $[78,79]$ as sensing materials due to their hygroscopic natures. These sensors have been used for many applications including human breath analysis and real-time tracking of plant transpiration due to their rapid responses to humidity variations, high sensitivity, and high stability $[78,79]$. Recently, Tour et al. have improved the spatial resolution $(\sim 12 \mu \mathrm{m})$ of the LIG patterns on PI by using a visible $405 \mathrm{~nm}$ laser and have fabricated a miniaturized humidity sensor, which can precisely analyze the PI conductivities at different relative humidity $(\mathrm{RH})$ levels [12]. The gap between two LIG electrodes is only $\sim 7 \mu \mathrm{m}$, which ensures a rapid response time $(250 \mathrm{~ms})$ for human breath analysis. In addition, bulk absorption-based microwave humidity sensing has also been demonstrated by integrating LIG with a planar microwave resonator [80]. The resulted device shows linear responses to a wide range of humidity levels (10\%-95\% RH), low hysteresis, and good long-term stability.

\subsubsection{Electrophysiological sensors}

Point-of-care wearable devices that can achieve continuous and real-time electrophysiological signal recording is of significance for mobile health monitoring and human-machine interface [74, 81, 82]. LIG-based wearable sensors have been developed and can be laminated on various locations of human bodies to record different electrophysiological signals, such as ECG, electromyograms (EMG), and electroencephalograms (EEG) $[35,37,83]$. As shown in Figs. 7(a)-7(f), our research group has recently developed breathable wearable electrophysiological sensors, comprising LIG sensing elements transfer-printed on porous PDMS supporting substrates [37]. The alpha rhythm in the EEG spectrum (Figs. 7(b) and 7(c)), characteristic P wave, QRS complex, and T wave in the ECG signals (Figs. 7(d) and $7(\mathrm{e})$ ), and the muscle activities indicated by the EMG signals (Fig. 7(f)) are clearly distinguishable. Such sensing capabilities have also been demonstrated by nonporous PDMS- and textile-based LIG electrophysiological sensors [35, 83]. Besides, LIG-based implantable cortical microelectrodes exhibited high potentials in neural sensing and stimulations, largely because of low impedance, high charge injection capacity, and high flexibility [58]. As shown in Fig. 7(g), in vivo 


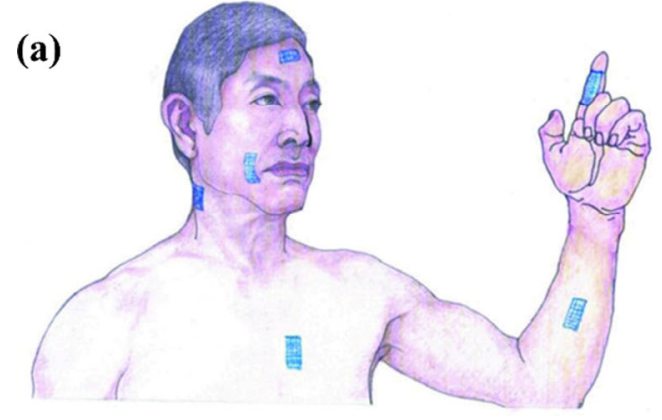

(d)
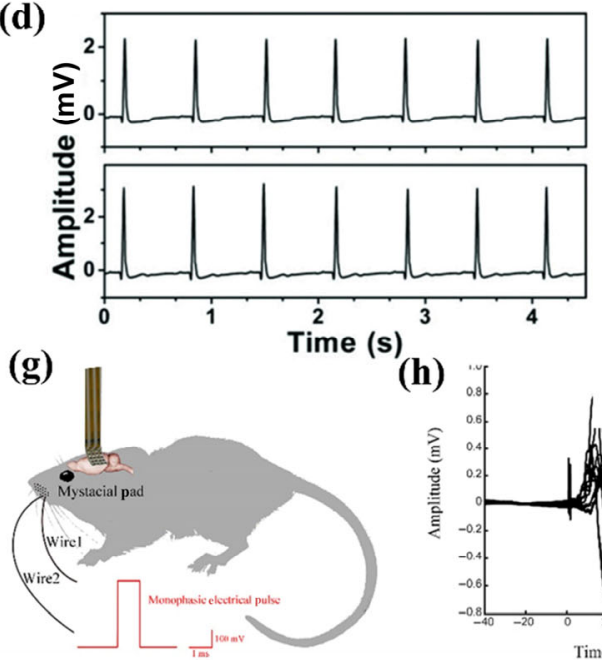

(h)

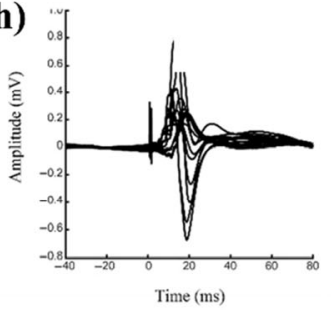

(b)

(e)
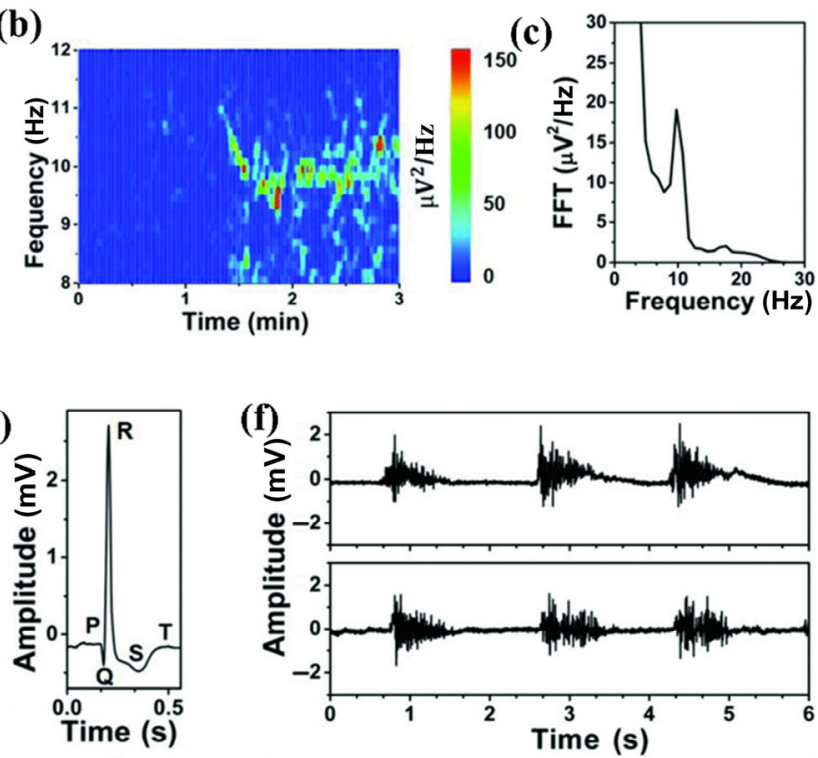

(i)

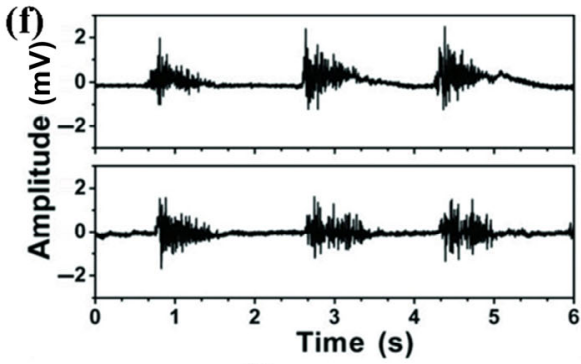

(j)

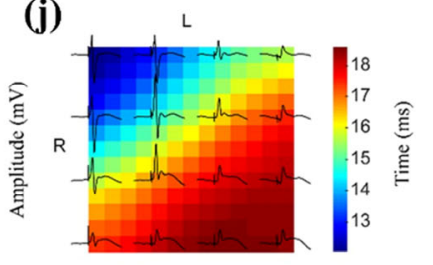

Figure 7 (a) Schematic illustration of LIG-based devices for electrophysiological signal recording. (b) and (c) Recorded EEG signals. (d) and (e) Recorded ECG signals. (f) Recorded EMG signals. Reproduced with permission from Ref. [37], (c) WILEY-VCH Verlag GmbH \& Co. KGaA, Weinheim 2018. (g) Schematic illustration of SEPs recording setup. (h) Recorded SEPs signals. The amplitude (i) and (j) latency of the first positive peak of the SEPs. Reproduced with permission from Ref. [58], (c) Lu, Y. et al. 2016.

experiments were conducted on adult rat models. When electrical stimulations are applied, a 16-LIG-microelectrode array provides high-fidelity recording of somatosensoryevoked potentials (SEPs) at the pial surface of barrel cortex (Fig. 7(h)). The variations of the amplitude and latency from the first positive peak of the SEPs are also clearly observed (Figs. 7(i) and 7(j)) [58].

\subsection{LIG-based biochemical sensors}

\subsubsection{Gas sensors}

Graphene has been widely used for gas detections including $\mathrm{NO}_{2}, \mathrm{NH}_{3}, \mathrm{H}_{2} \mathrm{~S}$, and $\mathrm{H}_{2}$ due to electrical signal variations caused by the change of local carrier concentrations in response to the adsorption and desorption of gas molecules [84]. LIG has porous structures and large surface areas, which provide a large amount of surface active sites for the interaction with gaseous compounds. By taking advantage of thermal conductivities of different gases, Tour et al. have developed a LIG-based gas sensor, as illustrated in Fig. 8(a) [48]. Localized heat is generated in the sensing filament (line thickness: $\sim 57 \mu \mathrm{m}$ ) when a bias voltage is applied due to the large resistance of the thin LIG line. The generated heat is subsequently dissipated to the surrounding environment via convective heat loss. The materialdependent heat transfer coefficient of various gas analytes, together with the temperature-dependent electrical feature of LIG, allows to differentiate various gas molecules. That said, the LIG resistance change can therefore be used for gas detections, as evidenced in Fig. 8(b). In this study, various gases including air, helium, oxygen, and carbon dioxide are investigated. The obtained gas sensors exhibit good stability, good durability, and rapid response time (7-8 s) toward all gas species tested at room temperature. However, the sensitivity is relatively low (e.g., detection of limits $>100$ ppm). Recently, the semiconducting property of LIG has also been explored for gas sensing. For example, pristine LIG printed on textiles, serving as p-type semiconductor, has been used for $\mathrm{NO}_{2}$ sensing [35]. Oxidizing gas molecules (such as $\mathrm{NO}_{2}$ ) can withdraw electrons from LIG and the hole concentrations will therefore increase. The enabled LIG-based gas sensors fabricated on Kevlar textiles show good affinity and selectivity to $\mathrm{NO}_{2}$ over some interfering chemicals (e.g., chlorine, methanolic acid, and acetic acid). Besides, Cheng et al. have recently developed LIG-based wearable self-heating $\mathrm{NO}_{2}$ gas sensors [49]. Figure 8(c) shows their sensor fabrication process. Here, strategically designed LIG patterns are first made with $\mathrm{CO}_{2} \mathrm{IR}$ laser on PI and are then transfer-printed on elastomer substrates. Next, silver inks are selectively coated on LIG patterns to improve the conductivity and to generate required localized heat within the sensing areas. And, the reduced graphene oxide $(\mathrm{r}-\mathrm{GO}) / \mathrm{MoS}_{2}$ or other nanomaterials are coated onto pre-designed sensing areas to complete the device fabrication. At an optimized temperature $\left(60^{\circ} \mathrm{C}\right)$, the fabricated gas sensor exhibits excellent sensitivity to $\mathrm{NO}_{2}(\sim 1.5$ parts per billion) by leveraging the $\mathrm{p}-\mathrm{n}$ heterojunctions formed at the interfaces between r-GO and $\mathrm{MoS}_{2}$. Moreover, the enabled gas sensors also demonstrate good selectivity and decent stretchability, as illustrated in Fig. 8(d). In addition, the gas sensors based on pristine LIG and functionalized LIG decorated with $\mathrm{MoS}_{2}$ or palladium (Pd) nanoparticles have also been reported for the detections of $\mathrm{NH}_{3}, \mathrm{NO}_{2}$, and $\mathrm{H}_{2}[46,53,85]$.

\subsubsection{Biofluids biochemical sensors}

Since the discovery of LIG, its applications in biochemical 
(a)
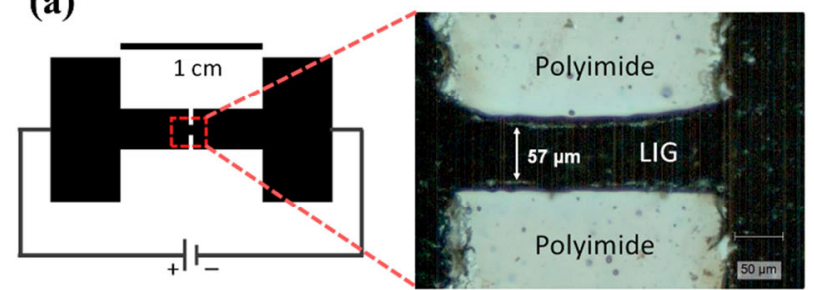

(b)

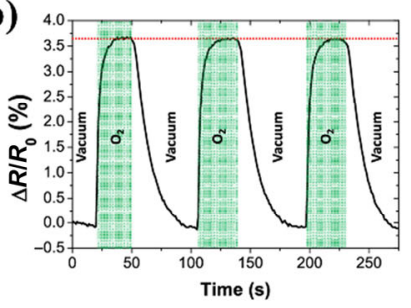

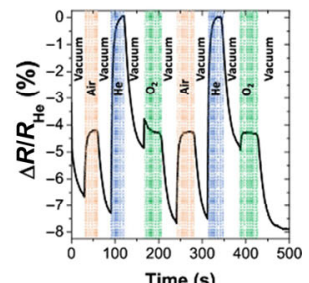

Time (s) (c)

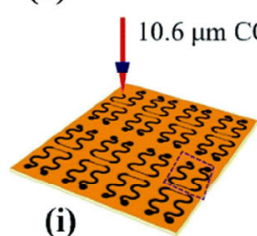

(i)

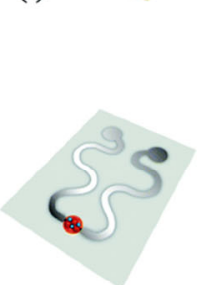

(iv)

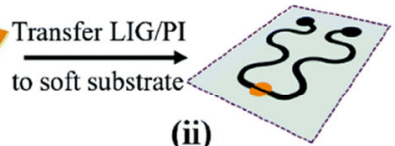

(ii)

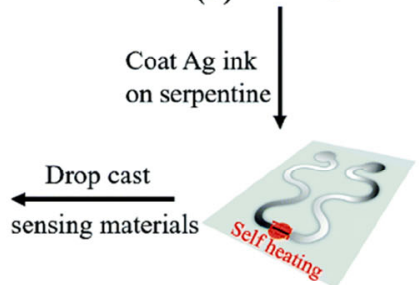

(iii) (d)
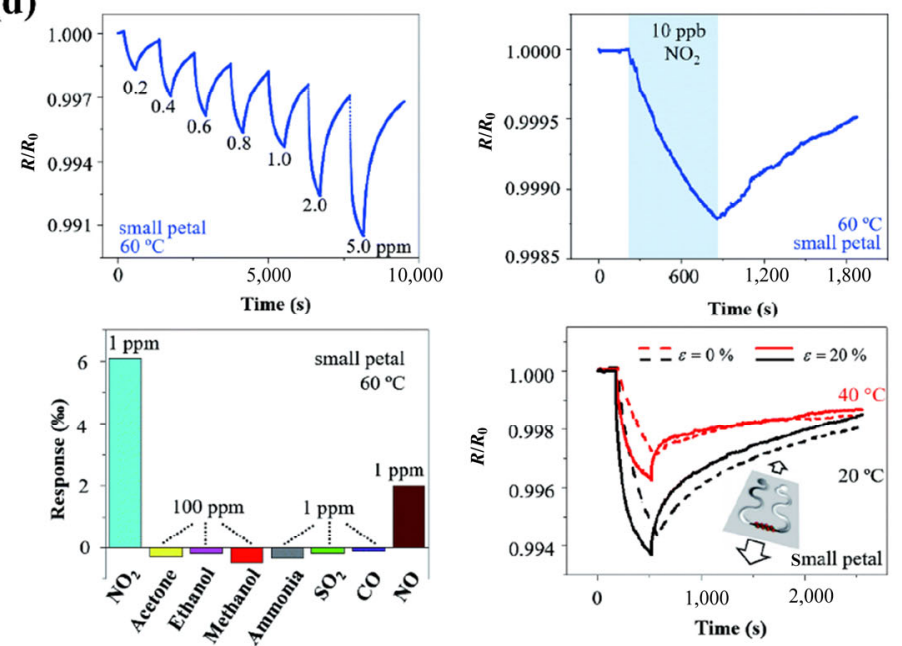

Figure 8 (a) The pristine LIG-based gas sensors. (b) Responses of the gas sensor to a variety of gases, showing high repeatability. Reproduced with permission from Ref. [48], @ American Chemical Society 2019. Schematic illustration of the fabrication process (c) and characterizations (d) of LIG-based self-heating wearable gas sensors. Reproduced with permission from Ref. [49], ๑ The Royal Society of Chemistry 2020.

sensing have attracted extensive research efforts due to its unique $3 \mathrm{D}$ porous structures, large surface areas, and rich surface defects, which are advantageous for electrochemicalenabled biosensing as electron transfer kinetics are significantly improved [86]. Currently, LIG-enabled biochemical sensors have been widely explored for applications in food safety, water and air quality, and biofluids (e.g., sweat, urine, saliva) analysis. Moreover, their performance usually outperforms the devices made of other types of graphene-based materials due to the $3 \mathrm{D}$ porous structures and rich surface defects of LIG. The biochemical sensors that use pristine LIG and functionalized LIG as the sensing elements are comprehensively summarized in Table 3, where the targeted analytes, LIG surface modifications, and sensing methods are included. The analytes mainly include electrolytes, small molecules, pathogens, nucleic acids, and immunology. As several recent reviews have detailed some of the related work $[18,19]$, we only highlight the most recent studies and some representative examples in this section.

Benefiting from large surface areas and rich surface defects induced during the laser scribing process, pristine LIG-based devices have been widely explored for detections of small molecules [50, 86-90] and nucleic acids [91] in biofluids (e.g., sweat, urine, saliva). For instance, $\mathrm{Xu}$ et al. have developed the electrochemical neurotransmitter sensors based on grass-like LIG, which can selectively detect dopamine (DA), epinephrine (EP) and norepinephrine (NE) using the differential pulse voltammetry (DPV) method [87]. The in vitro analysis of human urine samples indicates the applicability to real biofluid analysis. By integrating the sample collection, real-time analysis, and wireless data transmission into one single platform, Gao et al. have developed LIG-based wearable sweat sensors for concurrent detections of uric acid (UA) and tyrosine (Tyr), which are usually associated with some human diseases such as gout and metabolic disorders [50]. Figure 9 provides the schemes and characterizations of LIG-based wearable sweat UA and Tyr sensors. The enabled device comprises LIG working and counter electrodes and Ag-LIG reference electrodes, which can selectively oxidize UA and Tyr at specific potentials. The enabled sweat biosensors with optimized synthetic conditions exhibit high sensitivities (3.50 and $\left.0.61 \mu \mathrm{A} /\left(\mu \mathrm{M} \cdot \mathrm{cm}^{2}\right)\right)$ and low detection limits $(0.74$ and $3.6 \mu \mathrm{M})$ for UA and Tyr, respectively (Figs. 9(e) and 9(f)). Moreover, the oxidation potentials for different analytes are intrinsically distinct, allowing their highly selective detections in the presence of some common interfering molecules, such as glucose (Glu), ascorbic acid (AA), DA and urea (Figs. 9(g)-9(i)). Furthermore, Fig. 9(j) demonstrates that LIG-based electrodes (LEGE) exhibit superior electrochemical activities over conventional electrode materials, such as commercial glassy carbon (GCE), screen-printed carbon (SPE), and gold electrodes (AuE). Thus, LIG-based biochemical sensors demonstrate better performances than the devices made of the aforementioned conventional materials, in terms of the analysis of sweat and saliva biochemicals (Figs. 9(k) and 9(l)). In this study, in vivo demonstrations are also provided for gout management, indicating promising applications of LIG-based biochemical sensors for personalized sweat analysis. In addition, the ability of LIG-based biochemical sensors to detect other analytes in biofluids, such as sodium sulfate, hydrazine, miRNA, and lead $(\mathrm{Pb})$, have also been recently reported [88-91].

Furthermore, the LIG functionalized with other materials has been explored for the detections of a broad spectrum of analytes spanning from ions, small molecules, pathogens, nucleic acids, to immunology. Surface functionalization typically endows the pristine LIG with enhanced conductivity, improved selectivity, and increased sensitivity. Yu et al. have developed flexible electronic tongue systems for multi-flavor detections via modifying LIG sensor arrays with gold nanoparticles (AuNPs), r-GO, and polyaniline (PANI) [92]. In addition to improving conductivity, AuNPs can also enhance the local electromagnetic field [93], thereby improving selectivity. The resulting biochemical 
Table 3 LIG-based biochemical sensors

\begin{tabular}{|c|c|c|c|c|}
\hline Category & Analyte & Modification & Sensing modality & Ref. \\
\hline \multirow{5}{*}{ Ions/elements } & $\mathrm{NH}_{4}{ }^{+}$and $\mathrm{K}^{+}$ & $\mathrm{NH}_{4}^{+}, \mathrm{K}^{+}$ionophores & Potentiometry & {$[115]$} \\
\hline & $\mathrm{NH}_{4}{ }^{+}$and $\mathrm{NO}_{3}{ }^{-}$ & $\mathrm{NH}_{4}{ }^{+}, \mathrm{NO}_{3}{ }^{-}$ionophores & Potentiometry & {$[13]$} \\
\hline & $\mathrm{Cu}^{2+}$ & $\mathrm{CdS}, \mathrm{PbS}$ & Photoelectrochemical analysis & [23] \\
\hline & $\mathrm{Cd}$ and $\mathrm{Pb}$ & Polyaniline, polyvinylpyrrolidone & Square wave anodic stripping voltammetry & {$[116]$} \\
\hline & $\mathrm{Pb}$ & None & Anodic stripping voltammetry & [90] \\
\hline \multirow{20}{*}{ Small molecules } & $\mathrm{AA}, \mathrm{UA}$ and $\mathrm{DA}$ & Pt nanoparticles & Differential pulse voltammetry & {$[86]$} \\
\hline & Glucose & Electrocatalyst, CdS & Photoelectrochemical analysis & {$[117]$} \\
\hline & Glucose & Glucose oxidase & $\mathrm{CV}$, chronoamperometry & {$[25,83,100]$} \\
\hline & Glucose & $\mathrm{Cu}$ Pt nanoparticles & Chronoamperometry & {$[118]$} \\
\hline & Glucose & $\mathrm{Cu}$ nanocubes & Chronoamperometry & [119] \\
\hline & $\mathrm{H}_{2} \mathrm{O}_{2}$ & Pt nanoparticles & Amperometry & {$[120,121]$} \\
\hline & $\mathrm{H}_{2} \mathrm{O}_{2}$ & Ag nanoparticles & Chronoamperometry & {$[122]$} \\
\hline & Urea & Urease & $\mathrm{pH}$ indicator & {$[123]$} \\
\hline & Thrombin & Aptamer & Differential pulse voltammetry & [98] \\
\hline & Thrombin & Aptamer, liposomes & Electrochemical impedance spectroscopy & {$[124]$} \\
\hline & Bisphenol A & Aptamer & Capacitive sensing & [96] \\
\hline & Cortisol & Anti-cortisol antibody & Chronoamperometry & {$[101]$} \\
\hline & Dopamine & Poly(3,4-ethylenedioxythiophene) & Differential pulse voltammetry & {$[125]$} \\
\hline & Dopamine & $\mathrm{Pt}, \mathrm{Au}$ nanoparticles & Differential pulse voltammetry & [126] \\
\hline & Biogenic amines & Diamine oxidase & Chronoamperometry & {$[127]$} \\
\hline & UA and tyrosine & None & Differential pulse voltammetry & {$[50]$} \\
\hline & Sugar/salt/vinegar & $\mathrm{Au}, \mathrm{rGO}$, and PANI & Impedance measurement & [92] \\
\hline & Sodium sulfate & None & Impedance measurement & [88] \\
\hline & Hydrazine & None & Cyclic voltammetry & [89] \\
\hline & $\mathrm{DA}, \mathrm{EP}$, and NE & None & Differential pulse voltammetry & {$[87]$} \\
\hline \multirow{2}{*}{ Pathogen } & E. coli. & Anti-E. coli antibody & Electrochemical impedance spectroscopy & {$[128]$} \\
\hline & Salmonella enterica & Anti-Salmonella antibody & Electrochemical impedance spectroscopy & [99] \\
\hline \multirow{2}{*}{ Nucleic acid } & miRNA & None & Differential pulse voltammetry & {$[91]$} \\
\hline & SARS-CoV-2 & Antigens and antibodies & Differential pulse voltammetry & {$[102]$} \\
\hline \multirow{3}{*}{ Immunology } & Chloramphenicol & Molecularly imprinted polymer & Electrochemical impedance spectroscopy & [129] \\
\hline & Acetylcholinesterase inhibitor & $\mathrm{TiO}_{2}$ nanoparticles & Photoelectrochemical analysis & {$[130]$} \\
\hline & AA, amoxicillin & Molecularly imprinted polymer & Square wave voltammetry & [97] \\
\hline
\end{tabular}

sensors with various surface functionalizations exhibit different affinities to a particular type of molecules. That said, various responses toward salt, sugar and vinegar can be enabled by LIG sensors through different surface functionalizations, thus allowing the formation of a complex flavor fingerprint. Similar detection mechanisms, which mimic mammalian olfactory systems, were also reported for multi-component gas sensing $[94,95]$. By leveraging the principal component analysis (PCA) technique, salty, sweet and sour flavors can be discriminated and the concentrations of each analyte can be reliably predicted, as demonstrated in Fig. 10(a) [92].

In contrast to semiselective electronic tongue systems, surface modifications have also been explored to endow the pristine LIG with highly selective detections toward specific biochemicals and even bacteria. Functional groups usually involve antibodies, enzymes, aptamers, and molecularly imprinted polymers [83, 96-101]. For example, Soares et al. have reported LIG-based electrochemical immunosensors for detections of Salmonella enterica, as schematically illustrated in Fig. 10(b) [99]. Here, LIG patterns are first generated using a $\mathrm{CO}_{2}$ IR laser.
Then, Salmonella antibodies are subsequently anchored on the porous LIG working electrode via carbodiimide cross-linking chemistry. The specific adhesion of bacterial cells onto the functionalized LIG working electrodes hinders the electrolyte access when evaluated through the electrochemical impedance spectroscopy (EIS) method, thus leading to the increased charge transfer resistance $\left(R_{\mathrm{ct}}\right)$. The correlations between the changes of $R_{\mathrm{ct}}$ and bacterial concentrations can be used to predict the analyte concentrations. The calibration curve exhibits good linearity spanning from 25 to $10^{5}$ colony-forming unit (CFU)/mL good sensitivity, and low detection limits $(\sim 10 \mathrm{CFU} / \mathrm{mL})$. Moreover, the selectivity is also evaluated in the presence of five different interferent bacteria strains, indicating the high applicability to rapid, low-cost, high-selectivity pathogen detections in food processing facilities. Additionally, biochemical sensors based on functionalized LIG have also been explored for the detection of many other analytes in biofluids, such as ions, small molecules, pathogens, nucleic acids, and immunology. Detailed information is provided in Table 3. It is worth noting that, most recently, biochemical sensors based on the antibody- 
(a)

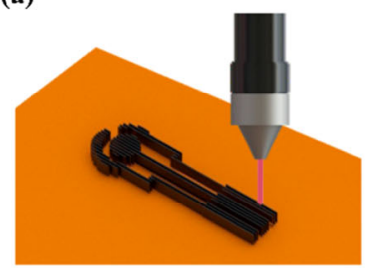

(e)
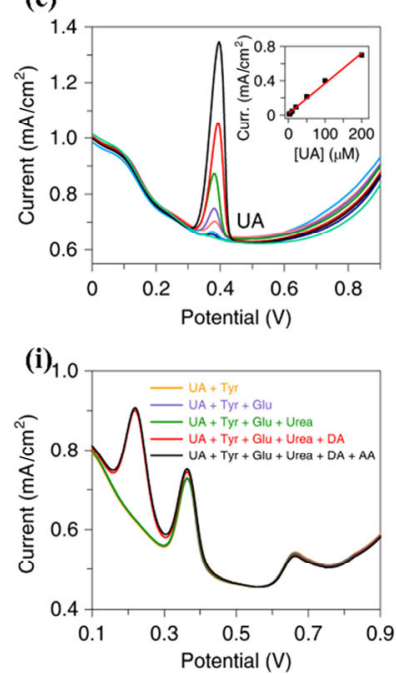

(b)

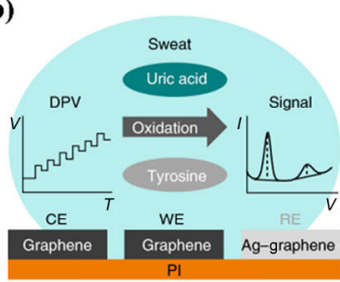

(f)

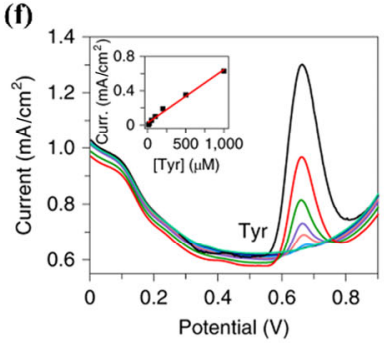

(j)

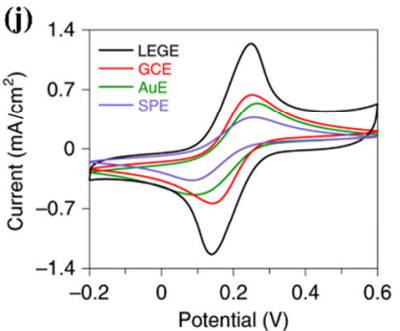

(c)

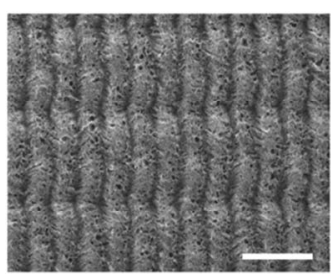

(g)

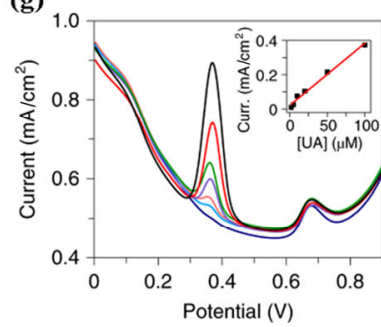

(k)

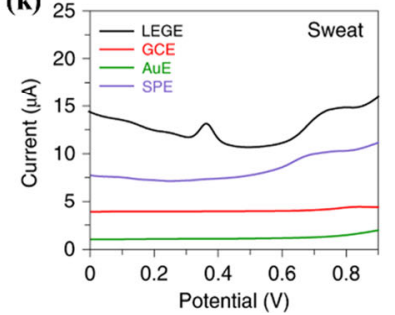

(d)

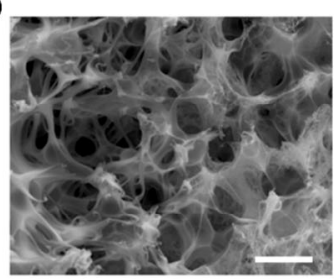

(h)

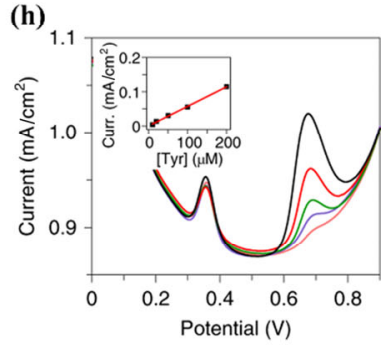

(1)

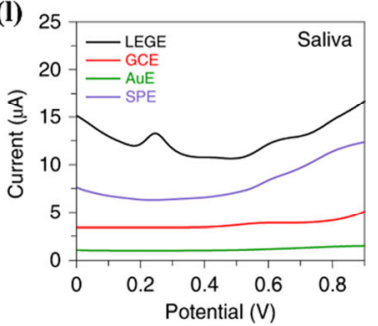

Figure 9 (a) Schematic illustration of the fabrication of LIG-based wearable biochemical sweat sensors. (b) Diagram of a three-electrode sweat UA and Tyr sensors. CE, counter electrode; WE, working electrode; RE, reference electrode. (c) and (d) SEM images of LIG for sweat sensors. Scale bars, $200 \mu \mathrm{m}$ (c) and $3 \mu \mathrm{m}$ (d). UA (e) and Tyr (f) detections using the DPV method. (g) and (h) Simultaneous and selective detections of UA and Tyr. (i) Selectivity tests in the presence of various interfering molecules. (j) Comparisons of CV scans with different electrode materials. DPV signals in the raw sweat (k) and saliva (l) samples measured with different electrodes. Reproduced with permission from Ref. [50], () Yang, Y. R. et al. 2020.

modified LIG have been developed for COVID-19 diagnosis in response to the pandemic outbreak [102].

\subsection{Applications of LIG in energy harvesting and photodetection}

In addition to the aforementioned biophysical and biochemical sensors, LIG also shows its promises in many other applications, ranging from energy storage, catalysis, energy harvesting, to photodetection. The former two research directions are summarized in prior reviews $[15,16]$, we therefore only include the bioelectronics related applications of LIG in flexible energy harvesting and photodetection in this section. Recently, Tour et al. have developed LIG-based triboelectric nanogenerators (TENG), which use PI and cork as the carbon precursors [103]. In this study, the TENGs adopt the conductor-to-dielectric and metal-free dielectric-to-dielectric geometries, where LIG-PI composites serve as tribo-negative materials and aluminum or PU films serve as tribo-positive materials. Contact electrifications occur when the two ends are brought together, while electrical current flows caused by electrostatic induction occur through external loads when they are separated. The devices with conductor-to-dielectric structures exhibit open-circuit potentials of up to $3.5 \mathrm{kV}$, short-circuit currents of $\sim 60 \mu \mathrm{A}$, and peak power outputs of $\sim 2.4 \mathrm{~W} / \mathrm{m}^{2}$. This work provides a promising demonstration of LIG-based flexible and portable energy harvesting systems. Similar studies that use different carbon precursors have also been conducted to fabricate LIGbased TENGs [38, 104]. In addition, hygroelectric energy harvesters that rely on the LIG surface hydrophilicity have been reported by Ye et al. [56]. Leveraging tunable surface properties enabled by adjusting lasing parameters, LIG with gradient hydrophilicity (i.e., various oxidized conditions) is generated on the PI substrate. LIG with higher affinity to water absorbs more water molecules and subsequently releases protons when exposed to water vapors, leading to the formation of a proton gradient. This gradient distribution can cause the current flow through an external circuit, as often seen in many GO-based moisture-electric energy harvesters [105-107].

Besides, Lin et al. have developed monolithic and flexible UV photodetectors by laser-assisted in situ formation of the functionalized LIG decorated with $\mathrm{ZnS} / \mathrm{SnO}_{2}$ nanoparticles [108]. The one-step laser scribing process not only leads to a good interface between the LIG electrodes and semiconductive nanomaterials, but reduces the total device thickness, which can improve the optoelectronic performance and enhance the reliability and flexibility. Recently, Chen et al. have fabricated a LIG-based capacitive proximity sensor using a UV laser, which can detect capacitance variations when an object approaches to the sensor surface [109].

\section{Applications of LIG in soft actuators and soft robots}

Recently, soft actuators, which can convert multiple stimulus (e.g., light, humidity, chemicals, magnetic field, electricity) to mechanical deformations in a controlled manner, have been widely studied due to their potential applications in soft robots and others [110]. Laser-assisted fabrication process provides simple, mask-free fabrications of LIG-based soft actuators with customizable designs that can be leveraged by emerging soft robotic techniques. In addition, the outstanding electrical and thermal conductivities and photothermal effects of LIG also show great promises in the development of soft actuators with different actuation mechanisms. Recently, Lin et al. have demonstrated LIG-based soft actuators that are responsive to multi-stimulus, including electricity, light, and chemicals [111]. 
(a)

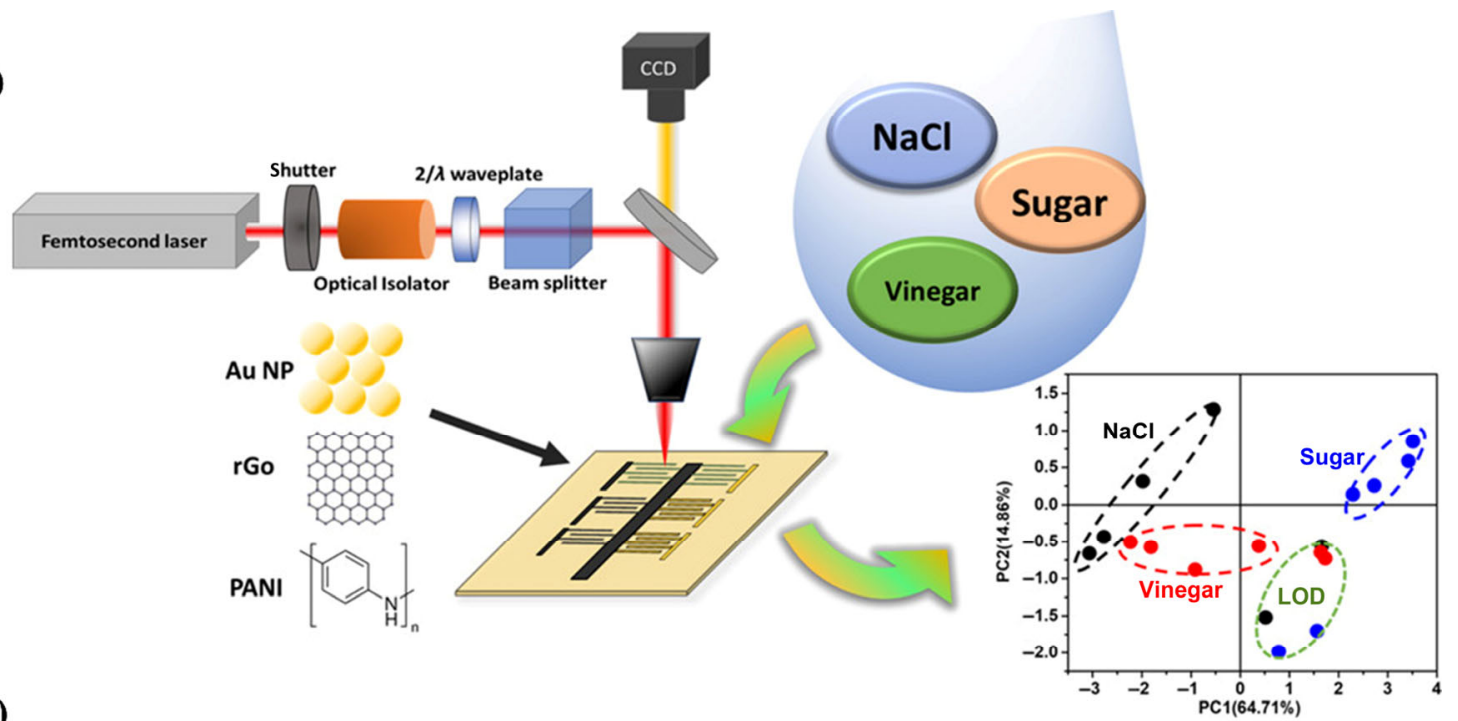

(b)
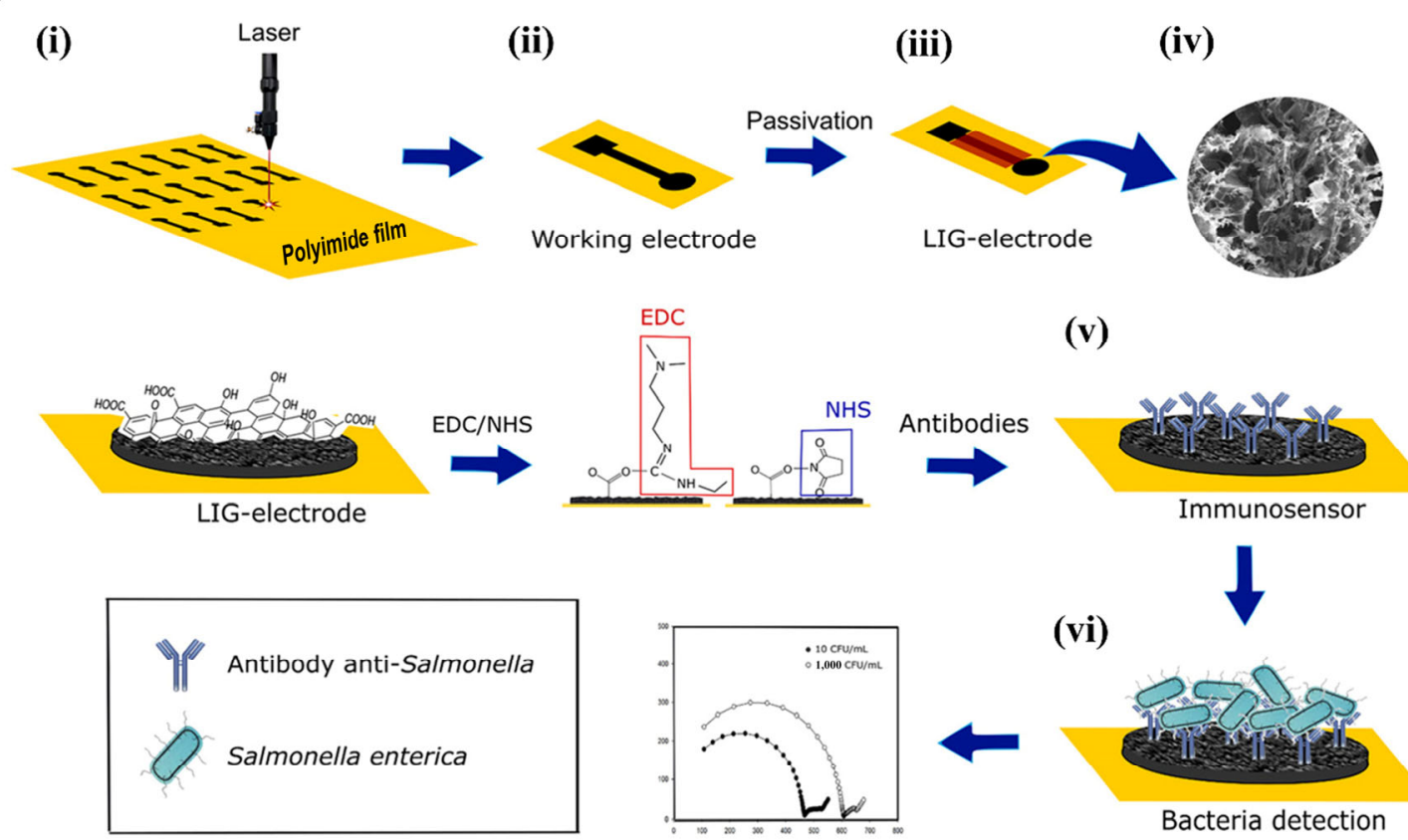

(v)
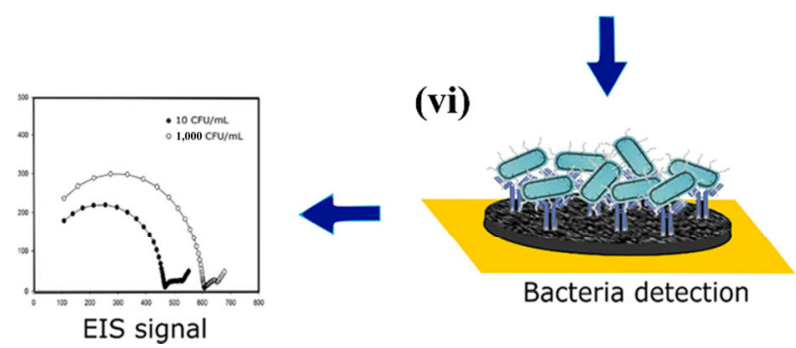

Figure 10 (a) Schematic illustration of the fabrication of AuNPs, r-GO and PNAI modified LIG sensor arrays and their applications for multi-flavor detections using principal component analysis. Reproduced with permission from Ref. [92], @ American Chemical Society 2018. (b) Schematic illustration of the fabrication, biofunctionalization and sensing of LIG-based immunosensors for Salmonella enterica detection using the EIS method. Reproduced with permission from Ref. [99], () American Chemical Society 2020.

Figure 11(a) illustrates the fabrication process. Here, LIG is first made on PI substrates and then transferred onto poly(vinylidene fluoride) (PVDF) films, followed by the attachment of the PI tape on LIG. The exceptional thermal expansion coefficient of PVDF over PI, together with the good photothermal property of LIG, allows both electrothermal and photothermal actuations of the fabricated devices. Besides, the resulting actuators can also respond to chemicals upon acetone or water vapor exposure by taking advantage of the swelling behavior of PVDF. Most recently, our research group has developed LIG-based electrothermal soft robotic hands that adopt a trilayer structure, comprising LIG joule heaters sandwiched between PI and PDMS layers [74]. As shown in Fig. 11(b), human hand gestures can be used to control the motions of soft robotic fingers by real-time EMG signals recording using on-skin wearable electrophysiological sensors. In another recent research, we have explored the applications of LIG-based soft electrothermal actuators in mechanically guided 3D assembly [112]. The
LIG-based soft actuators can be used to construct programmable 3D structures through reversibly controlled, global folding and/or local bending. A variety of complex reconfigurable 3D structures can be realized by further incorporating origami and kirigami designs and separately controlling addressable LIG soft actuators. As illustrated in Fig. 11(c), the 3D structures, which resemble flower stamen, epiphytic orchid and foldable quatrefoil, can be made and sequentially reshaped into various programmed geometries. Besides, light-driven LIG-based soft actuators that rely on photothermal Marangoni effect has been reported by Wang et al. [60]. And, the liquid surface tension generally decreases as the temperature increases, which allows the formation of the surface tension gradient through photothermal effects of LIG upon light irradiation. The light illumination therefore can remotely drive the translation and rotation motions of LIG-based soft actuators. By laser printing of various LIG patterns on adhesive PI tapes, a rich variety of adaptive 3D structures are demonstrated in this research. 
(a)
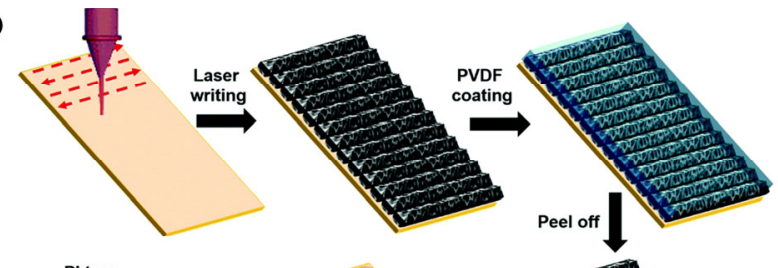

(b)

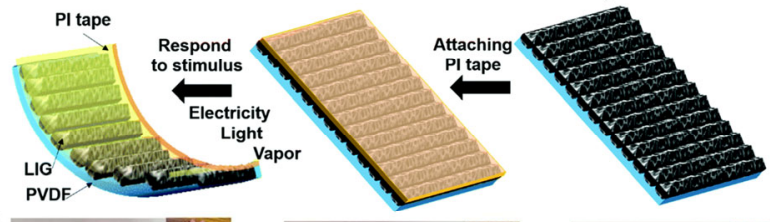

(c)
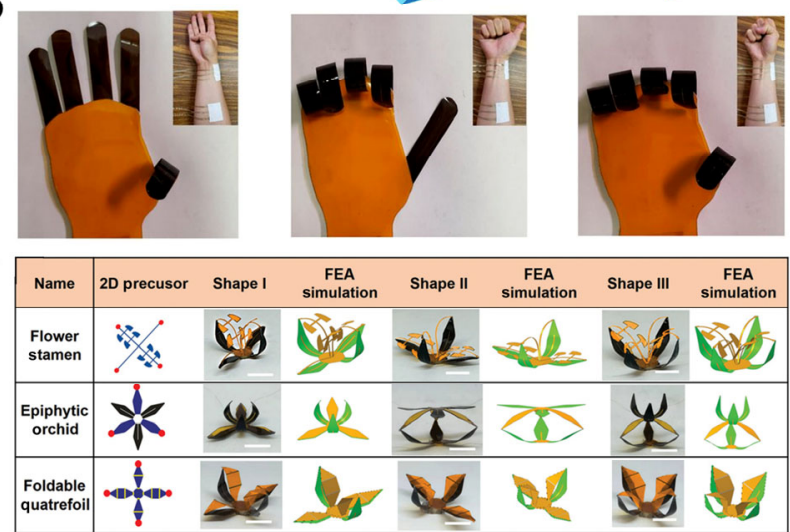

Figure 11 (a) Schematic illustration of the fabrication of a multi-responsive LIG-based soft actuator. Reproduced with permission from Ref. [111], () The Royal Society of Chemistry 2018. (b) Photographs of a LIG-based soft robotic hand, which can be controlled by human gestures via real-time EMGs recording. Reproduced with permission from Ref. [74], @ Xu, Y. D. et al. 2020. (c) Three representative examples of reconfigurable 3D assembly enabled by LIG-based electrothermal soft actuators. Scale bars, $1 \mathrm{~cm}$. Reproduced with permission from Ref. [112], ๑ WILEY-VCH Verlag GmbH \& Co. KGaA, Weinheim 2020.

\section{Conclusion and perspectives}

\subsection{Synthesis of LIG}

Since the discovery of LIG in 2014, significant advancements have been achieved in both fundamental synthesis and practical applications. Notably, the laser-assisted process can enable facile, mask-free, large-area, inexpensive, customizable, and miniaturized patterning of LIG in a programmable manner on versatile flexible carbonaceous substrates at ambient conditions, which are advantageous over other graphene-based materials especially for emerging applications of bioelectronics and soft actuators. Another unique feature is that the LIG's porosity, morphology, composition, and electrical conductivity can be tuned for different applications. Also, the obvious progress of porous structures engineering of LIG and its supporting substrates has been achieved. And, roll-to-roll production $[15,38,69]$ and additive manufacturing of LIG (i.e., 3D printing) $[113,114]$ have been explored. In addition, structural engineering (e.g., kirigami patterns, 3D assembly) have been used to improve the stretchability of LIG-based devices. Besides, recent studies have illustrated the LIG's outstanding antibacterial property and biocompatibility, which are beneficial to its applications in biointegrated electronic devices.

\subsection{Applications of LIG}

Due to the unique features, pristine and functionalized LIG can find broad applications in bioelectronic devices and soft actuators. Currently, LIG-based materials have been widely used to construct various wearable biophysical sensors, such as strain sensors, pressure sensors, temperature sensors, hydration sensors, humidity sensors, and electrophysiological sensors, which can provide continuous monitoring of motions and dynamics of the body's status. In addition, its large surface areas and rich surface defects generated during laser scribing make LIG an ideal candidate over other graphene-based materials for biochemical sensing. A number of analytes, such as electrolytes, small molecules, nucleic acids, pathogens, and immunology, can be detected with biochemical sensors made of pristine LIG and/or functionalized LIG, which can be employed for human disease diagnosis, food safety, and water, air and soil quality examination.

\subsection{Challenges and outlook}

Despite recent advancements in LIG synthesis and applications, some critical challenges still remain. Specifically, the materials and chemistry aspects are still the key for the applications of LIG in bioelectronic devices and soft actuators. While a decent electrical conductivity $(\sim 10 \Omega / \mathrm{sq})$ has been achieved in LIG, further improvement is still needed for bioelectronic applications, especially where LIG will serve as interconnects and antennas. Besides, high-quality LIG are often obtained on thermoplastic polymers with high melting points (e.g., PI), which usually exhibit high stiffens and poor stretchability as compared to soft elastomers. Thus, an additional transfer process is needed to make LIG-based devices on soft elastic substrates. From this perspective, it is highly desirable to explore fabrication strategies to make high-quality LIG and other functional materials on elastic substrates. In addition, the reproducibility, stability, uniformity, and durability of LIG-based bioelectronic devices have been rarely studied by in vivo testing. More research efforts are therefore needed in the future. Lastly, the research of LIG-based soft actuators and soft robots is still in an infant stage. There should be many appealing opportunities in this research direction. Also, the integration with artificial intelligence and machine-learning algorithms may bring more opportunities for the controlled synthesis of LIG with desired properties, and the design and fabrications of LIG-based soft devices with required modalities.

\section{Acknowledgements}

Z. Y. acknowledges the financial support from the NSF grant (ECCS-1917630) and University of Missouri-Columbia startup fund.

\section{References}

[1] Geim, A. K. Graphene: Status and prospects. Science 2009, 324, 1530-1534.

[2] Chen, Z. P.; Ren, W. C.; Gao, L. B.; Liu, B. L.; Pei, S. F.; Cheng, H. M. Three-dimensional flexible and conductive interconnected graphene networks grown by chemical vapour deposition. Nat. Mater. 2011, 10, 424-428.

[3] Zhang, Z. K.; Du, J. H.; Zhang, D. D.; Sun, H. D.; Yin, L. C.; Ma, L. P.; Chen, J. S.; Ma, D. G.; Cheng, H. M.; Ren, W. C. Rosin-enabled ultraclean and damage-free transfer of graphene for large-area flexible organic light-emitting diodes. Nat. Commun. 2017, 8, 14560.

[4] Novoselov, K. S.; Geim, A. K.; Morozov, S. V.; Jiang, D.; Zhang, Y.; Dubonos, S. V.; Grigorieva, I. V.; Firsov, A. A. Electric field effect in atomically thin carbon films. Science 2004, 306, 666-669.

[5] Li, X. S.; Cai, W. W.; An, J.; Kim, S.; Nah, J.; Yang, D. X.; Piner, R.; Velamakanni, A.; Jung, I.; Tutuc, E. et al. Large-area synthesis of high-quality and uniform graphene films on copper foils. Science 2009, 324, 1312-1314.

[6] Chua, C. K.; Pumera, M. Chemical reduction of graphene oxide: A synthetic chemistry viewpoint. Chem. Soc. Rev. 2014, 43, 291-312. 
[7] Luong, D. X.; Bets, K. V.; Algozeeb, W. A.; Stanford, M. G.; Kittrell, C.; Chen, W. Y.; Salvatierra, R. V.; Ren, M. Q.; McHugh, E. A.; Advincula, P. A. et al. Gram-scale bottom-up flash graphene synthesis. Nature 2020, 577, 647-651.

[8] El-Kady, M. F.; Kaner, R. B. Direct laser writing of graphene electronics. ACS Nano 2014, 8, 8725-8729.

[9] El-Kady, M. F.; Strong, V.; Dubin, S.; Kaner, R. B. Laser scribing of high-performance and flexible graphene-based electrochemical capacitors. Science 2012, 335, 1326-1330.

[10] Lin, J.; Peng, Z. W.; Liu, Y. Y.; Ruiz-Zepeda, F.; Ye, R. Q.; Samuel, E. L. G.; Yacaman, M. J.; Yakobson, B. I.; Tour, J. M. Laser-induced porous graphene films from commercial polymers. Nat. Commun. 2014, 5, 5714 .

[11] Carvalho, A. F.; Fernandes, A. J. S.; Leitão, C.; Deuermeier, J.; Marques, A. C.; Martins, R.; Fortunato, E.; Costa, F. M. Laserinduced graphene strain sensors produced by ultraviolet irradiation of polyimide. Adv. Funct. Mater. 2018, 28, 1805271.

[12] Stanford, M. G.; Zhang, C.; Fowlkes, J. D.; Hoffman, A.; Ivanov, I. N.; Rack, P. D.; Tour, J. M. High-resolution laser-induced graphene. Flexible electronics beyond the visible limit. ACS Appl. Mater. Interfaces 2020, 12, 10902-10907.

[13] Garland, N. T.; McLamore, E. S.; Cavallaro, N. D.; Mendivelso-Perez, D.; Smith, E. A.; Jing, D. P.; Claussen, J. C. Flexible laser-induced graphene for nitrogen sensing in soil. ACS Appl. Mater. Interfaces 2018, 10, 39124-39133.

[14] You, R.; Liu, Y. Q.; Hao, Y. L.; Han, D. D.; Zhang, Y. L.; You, Z. Laser fabrication of graphene-based flexible electronics. $A d v$. Mater. 2020, 32, 1901981.

[15] Ye, R. Q.; James, D. K.; Tour, J. M. Laser-induced graphene. Acc. Chem. Res. 2018, 51, 1609-1620.

[16] Ye, R. Q.; James, D. K.; Tour, J. M. Laser-induced graphene: From discovery to translation. Adv. Mater. 2019, 31, 1803621.

[17] Liu, Y. Q.; Chen, Z. D.; Mao, J. W.; Han, D. D.; Sun, X. Laser fabrication of graphene-based electronic skin. Front. Chem. 2019, 7, 461.

[18] Huang, L. B.; Su, J. J.; Song, Y.; Ye, R. Q. Laser-induced graphene: En route to smart sensing. Nano-Micro Lett. 2020, 12, 157.

[19] Wan, Z. F.; Nguyen, N. T.; Gao, Y. S.; Li, Q. Laser induced graphene for biosensors. Sustain. Mater. Technol. 2020, 25, e00205.

[20] Vashisth, A.; Kowalik, M.; Gerringer, J. C.; Ashraf, C.; Van Duin, A. C. T.; Green, M. J. ReaxFF simulations of laser-induced graphene (LIG) formation for multifunctional polymer nanocomposites. ACS Appl. Nano Mater. 2020, 3, 1881-1890.

[21] Lamberti, A.; Serrapede, M.; Ferraro, G.; Fontana, M.; Perrucci, F.; Bianco, S.; Chiolerio, A.; Bocchini, S. All-SPEEK flexible supercapacitor exploiting laser-induced graphenization. $2 D$ Mater. 2017, 4, 035012.

[22] Singh, S. P.; Li, Y. L.; Zhang, J. B.; Tour, J. M.; Arnusch, C. J. Sulfur-doped laser-induced porous graphene derived from polysulfoneclass polymers and membranes. ACS Nano 2018, 12, 289-297.

[23] Ge, L.; Hong, Q.; Li, H.; Liu, C. C.; Li, F. Direct-laser-writing of metal sulfide-graphene nanocomposite photoelectrode toward sensitive photoelectrochemical sensing. Adv. Funct. Mater. 2019, 29, 1904000.

[24] Yang, W. W.; Zhao, W.; Li, Q. S.; Li, H.; Wang, Y. L.; Li, Y. X.; Wang, G. Fabrication of smart components by $3 \mathrm{D}$ printing and laser-scribing technologies. ACS Appl. Mater. Interfaces 2020, 12, 3928-3935.

[25] Zhang, Z. C.; Song, M. M.; Hao, J. X.; Wu, K. B.; Li, C. Y.; Hu, C. G. Visible light laser-induced graphene from phenolic resin: A new approach for directly writing graphene-based electrochemical devices on various substrates. Carbon 2018, 127, 287-296.

[26] Cao, L. J.; Zhu, S. R.; Pan, B. H.; Dai, X. Y.; Zhao, W. W.; Liu, Y.; Xie, W. P.; Kuang, Y. B.; Liu, X. Q. Stable and durable laserinduced graphene patterns embedded in polymer substrates. Carbon 2020, 163, 85-94.

[27] Ye, R. Q.; Chyan, Y.; Zhang, J. B.; Li, Y. L.; Han, X.; Kittrell, C.; Tour, J. M. Laser-induced graphene formation on wood. Adv. Mater. 2017, 29, 1702211.

[28] Mahmood, F.; Zhang, H. W.; Lin, J.; Wan, C. X. Laser-induced graphene derived from Kraft lignin for flexible supercapacitors. ACS Omega 2020, 5, 14611-14618.
[29] Zhang, W. L.; Lei, Y. J.; Ming, F. W.; Jiang, Q.; Costa, P. M. F. J.; Alshareef, H. N. Lignin laser lithography: A direct-write method for fabricating 3D graphene electrodes for microsupercapacitors. $A d v$. Energy Mater. 2018, 8, 1801840.

[30] Edberg, J.; Brooke, R.; Hosseinaei, O.; Fall, A.; Wijeratne, K.; Sandberg, M. Laser-induced graphitization of a forest-based ink for use in flexible and printed electronics. npj Flex. Electron. 2020, 4 , 17.

[31] Chyan, Y.; Ye, R. Q.; Li, Y. L.; Singh, S. P.; Arnusch, C. J.; Tour, J. M. Laser-induced graphene by multiple lasing: Toward electronics on cloth, paper, and food. ACS Nano 2018, 12, 2176-2183.

[32] Zang, X. N.; Shen, C. W.; Chu, Y.; Li, B. X.; Wei, M. S.; Zhong, J. W.; Sanghadasa, M.; Lin, L. W. Laser-induced molybdenum carbide-graphene composites for $3 \mathrm{D}$ foldable paper electronics. Adv. Mater. 2018, 30, 1800062 .

[33] Zang, X. N.; Jian, C. Y.; Zhu, T. S.; Fan, Z.; Wang, W. L.; Wei, M. S.; Li, B. X.; Diaz, M. F.; Ashby, P.; Lu, Z. M. et al. Laser-sculptured ultrathin transition metal carbide layers for energy storage and energy harvesting applications. Nat. Commun. 2019, 10, 3112.

[34] Zang, X. N.; Tai, K. Y.; Jian, C. Y.; Shou, W.; Matusik, W.; Ferralis, N.; Grossman, J. C. Laser-induced tar-mediated sintering of metals and refractory carbides in air. ACS Nano 2020, 14, 10413-10420.

[35] Wang, H. M.; Wang, H. M.; Wang, Y. L.; Su, X. Y.; Wang, C. Y.; Zhang, M. C.; Jian, M. Q.; Xia, K. L.; Liang, X. P.; Lu, H. J. et al. Laser writing of Janus graphene/Kevlar textile for intelligent protective clothing. ACS Nano 2020, 14, 3219-3226.

[36] Rahimi, R.; Ochoa, M.; Yu, W. Y.; Ziaie, B. Highly stretchable and sensitive unidirectional strain sensor via laser carbonization. $A C S$ Appl. Mater. Interfaces 2015, 7, 4463-4470.

[37] Sun, B. H.; McCay, R. N.; Goswami, S.; Xu, Y. D.; Zhang, C.; Ling, Y.; Lin, J.; Yan, Z. Gas-permeable, multifunctional on-skin electronics based on laser-induced porous graphene and sugar-templated elastomer sponges. Adv. Mater. 2018, 30, 1804327.

[38] Li, J. T.; Stanford, M. G.; Chen, W. Y.; Presutti, S. E.; Tour, J. M. Laminated laser-induced graphene composites. ACS Nano 2020, 14, 7911-7919.

[39] Gao, Y.; Li, Q.; Wu, R. Y.; Sha, J.; Lu, Y. F.; Xuan, F. Z. Laser direct writing of ultrahigh sensitive SiC-based strain sensor arrays on elastomer toward electronic skins. Adv. Funct. Mater. 2019, 29, 1806786.

[40] Zhu, Y. S.; Cai, H. B.; Ding, H. Y.; Pan, N.; Wang, X. P. Fabrication of low-cost and highly sensitive graphene-based pressure sensors by direct laser scribing polydimethylsiloxane. ACS Appl. Mater. Interfaces 2019, 11, 6195-6200.

[41] Nair, V.; Yi, J.; Isheim, D.; Rotenberg, M.; Meng, L. Y.; Shi, F. Y.; Chen, X. Q.; Gao, X.; Prominski, A.; Jiang, Y. W. et al. Laser writing of nitrogen-doped silicon carbide for biological modulation. Sci. Adv. 2020, 6, eaaz2743.

[42] Davis, M. E. Ordered porous materials for emerging applications. Nature 2002, 417, 813-821.

[43] Miyamoto, A.; Lee, S.; Cooray, N. F.; Lee, S.; Mori, M.; Matsuhisa, N.; Jin, H.; Yoda, L.; Yokota, T.; Itoh, A. et al. Inflammation-free, gas-permeable, lightweight, stretchable on-skin electronics with nanomeshes. Nat. Nanotechnol. 2017, 12, 907-913.

[44] Tan, K. W.; Jung, B.; Werner, J. G.; Rhoades, E. R.; Thompson, M. O.; Wiesner, U. Transient laser heating induced hierarchical porous structures from block copolymer-directed self-assembly. Science 2015, 349, 54-58.

[45] Bergsman, D. S.; Getachew, B. A.; Cooper, C. B.; Grossman, J. C. Preserving nanoscale features in polymers during laser induced graphene formation using sequential infiltration synthesis. Nat. Commun. 2020, 11, 3636.

[46] Yan, W. H.; Yan, W. R.; Chen, T. D.; Xu, J. G.; Tian, Q.; Ho, D. Sizetunable flowerlike $\mathrm{MoS}_{2}$ nanospheres combined with laser-induced graphene electrodes for $\mathrm{NO}_{2}$ sensing. ACS Appl. Nano Mater. 2020, 3, 2545-2553.

[47] Peng, Z. W.; Ye, R. Q.; Mann, J. A.; Zakhidov, D.; Li, Y. L.; Smalley, P. R.; Lin, J.; Tour, J. M. Flexible boron-doped laser-induced graphene microsupercapacitors. ACS Nano 2015, 9, 5868-5875.

[48] Stanford, M. G.; Yang, K. C.; Chyan, Y.; Kittrell, C.; Tour, J. M. Laser-induced graphene for flexible and embeddable gas sensors. ACS Nano 2019, 13, 3474-3482. 
[49] Yang, L.; Yi, N.; Zhu, J.; Cheng, Z.; Yin, X. Y.; Zhang, X. Y.; Zhu, H. L.; Cheng, H. Y. Novel gas sensing platform based on a stretchable laser-induced graphene pattern with self-heating capabilities. J. Mater. Chem. A 2020, 8, 6487-6500.

[50] Yang, Y. R.; Song, Y.; Bo, X. J.; Min, J. H.; Pak, O. S.; Zhu, L. L.; Wang, M. Q.; Tu, J. B.; Kogan, A.; Zhang, H. X. et al. A laserengraved wearable sensor for sensitive detection of uric acid and tyrosine in sweat. Nat. Biotechnol. 2020, 38, 217-224.

[51] Tao, L. Q.; Tian, H.; Liu, Y.; Ju, Z. Y.; Pang, Y.; Chen, Y. Q.; Wang, D. Y.; Tian, X. G.; Yan, J. C.; Deng, N. Q. et al. An intelligent artificial throat with sound-sensing ability based on laser induced graphene. Nat. Commun. 2017, 8, 14579.

[52] Tehrani, F.; Beltrán-Gastélum, M.; Sheth, K.; Karajic, A.; Yin, L.; Kumar, R.; Soto, F.; Kim, J.; Wang, J.; Barton, S. et al. Laserinduced graphene composites for printed, stretchable, and wearable electronics. Adv. Mater. Technol. 2019, 4, 1900162.

[53] Zhu, J. X.; Cho, M.; Li, Y. T.; Cho, I.; Suh, J. H.; Del Orbe, D.; Jeong, Y.; Ren, T. L.; Park, I. Biomimetic turbinate-like artificial nose for hydrogen detection based on 3D porous laser-induced graphene. ACS Appl. Mater. Interfaces 2019, 11, 24386-24394.

[54] Ling, Y.; Zhuang, X. T.; Xu, Z.; Xie, Y. C.; Zhu, X. Y.; Xu, Y. D.; Sun, B. H.; Lin, J.; Zhang, Y. H.; Yan, Z. Mechanically assembled, three-dimensional hierarchical structures of cellular graphene with programmed geometries and outstanding electromechanical properties. ACS Nano 2018, 12, 12456-12463.

[55] Singh, S. P.; Li, Y. L.; Be'er, A.; Oren, Y.; Tour, J. M.; Arnusch, C. J. Laser-induced graphene layers and electrodes prevents microbial fouling and exerts antimicrobial action. ACS Appl. Mater. Interfaces 2017, 9, 18238-18247.

[56] Huang, L. B.; Xu, S. Y.; Wang, Z. Y.; Xue, K.; Su, J. J.; Song, Y.; Chen, S. J.; Zhu, C. L.; Tang, B. Z.; Ye, R. Q. Self-reporting and photothermally enhanced rapid bacterial killing on a laser-induced graphene mask. ACS Nano 2020, 14, 12045-12053.

[57] Zhong, H.; Zhu, Z. R.; Lin, J.; Cheung, C. F.; Lu, V. L.; Yan, F.; Chan, C. Y.; Li, G. J. Reusable and recyclable graphene masks with outstanding superhydrophobic and photothermal performances. ACS Nano 2020, 14, 6213-6221.

[58] Lu, Y. C.; Lyu, H.; Richardson, A. G.; Lucas, T. H.; Kuzum, D. Flexible neural electrode array based-on porous graphene for cortical microstimulation and sensing. Sci. Rep. 2016, 6, 33526.

[59] d'Amora, M.; Lamberti, A.; Fontana, M.; Giordani, S. Toxicity assessment of laser-induced graphene by zebrafish during development. J. Phys. Mater. 2020, 3, 034008.

[60] Wang, W.; Han, B.; Zhang, Y.; Li, Q.; Zhang, Y. L.; Han, D. D.; Sun, H. B. Laser-induced graphene tapes as origami and stick-on labels for photothermal manipulation via marangoni effect. Adv. Funct. Mater. 2021, 31, 2006179.

[61] Puetz, P.; Behrent, A.; Baeumner, A. J.; Wegener, J. Laser-scribed graphene (LSG) as new electrode material for impedance-based cellular assays. Sens. Actuators B: Chem. 2020, 321, 128443.

[62] Someya, T.; Bao, Z. N.; Malliaras, G. G. The rise of plastic bioelectronics. Nature 2016, 540, 379-385.

[63] Kim, D. H.; Lu, N. S.; Ma, R.; Kim, Y. S.; Kim, R. H.; Wang, S. D.; Wu, J.; Won, S. M.; Tao, H.; Islam, A. et al. Epidermal electronics. Science 2011, 333, 838-843.

[64] Chhetry, A.; Sharifuzzaman, M.; Yoon, H.; Sharma, S.; Xuan, X.; Park, J. Y. $\mathrm{MoS}_{2}$-decorated laser-induced graphene for a highly sensitive, hysteresis-free, and reliable piezoresistive strain sensor. ACS Appl. Mater. Interfaces 2019, 11, 22531-22542.

[65] Dallinger, A.; Keller, K.; Fitzek, H.; Greco, F. Stretchable and skin-conformable conductors based on polyurethane/laser-induced graphene. ACS Appl. Mater. Interfaces 2020, 12, 19855-19865.

[66] Chen, X. P.; Luo, F.; Yuan, M.; Xie, D. L.; Shen, L.; Zheng, K.; Wang, Z. P.; Li, X. D.; Tao, L. Q. A dual-functional graphene-based self-alarm health-monitoring E-skin. Adv. Funct. Mater. 2019, 29, 1904706.

[67] Luo, S. D.; Hoang, P. T.; Liu, T. Direct laser writing for creating porous graphitic structures and their use for flexible and highly sensitive sensor and sensor arrays. Carbon 2016, 96, 522-531.

[68] Tao, L. Q.; Wang, D. Y.; Tian, H.; Ju, Z. Y.; Liu, Y.; Pang, Y.; Chen, Y. Q.; Yang, Y.; Ren, T. L. Self-adapted and tunable graphene strain sensors for detecting both subtle and large human motions. Nanoscale 2017, 9, 8266-8273.
[69] Wang, Y. N.; Wang, Y.; Zhang, P. P.; Liu, F.; Luo, S. D. Laser-induced freestanding graphene papers: A new route of scalable fabrication with tunable morphologies and properties for multifunctional devices and structures. Small 2018, 14, 1802350.

[70] He, M. H.; Wang, Y. N.; Wang, S. R.; Luo, S. D. Laser-induced graphene enabled 1D fiber electronics. Carbon 2020, 168, 308-318.

[71] Kaidarova, A.; Khan, M. A.; Marengo, M.; Swanepoel, L.; Przybysz, A.; Muller, C.; Fahlman, A.; Buttner, U.; Geraldi, N. R.; Wilson, R. P. et al. Wearable multifunctional printed graphene sensors. npj Flex. Electron. 2019, 3, 15.

[72] Rahimi, R.; Ochoa, M.; Ziaie, B. Direct laser writing of porouscarbon/silver nanocomposite for flexible electronics. ACS Appl. Mater. Interfaces 2016, 8, 16907-16913.

[73] Tian, Q.; Yan, W. R.; Li, Y. Q.; Ho, D. Bean pod-inspired ultrasensitive and self-healing pressure sensor based on laser-induced graphene and polystyrene microsphere sandwiched structure. ACS Appl. Mater. Interfaces 2020, 12, 9710-9717.

[74] Xu, Y. D.; Sun, B. H.; Ling, Y.; Fei, Q. H.; Chen, Z. Y.; Li, X. P.; Guo, P. J.; Jeon, N.; Goswami, S.; Liao, Y. X. et al. Multiscale porous elastomer substrates for multifunctional on-skin electronics with passive-cooling capabilities. Proc. Natl. Acad. Sci. USA 2020, $117,205-213$.

[75] Shao, Q.; Liu, G.; Teweldebrhan, D.; Balandin, A. A. Hightemperature quenching of electrical resistance in graphene interconnects. Appl. Phys. Lett. 2008, 92, 202108.

[76] Bobinger, M. R.; Romero, F. J.; Salinas-Castillo, A.; Becherer, M.; Lugli, P.; Morales, D. P.; Rodríguez, N.; Rivadeneyra, A. Flexible and robust laser-induced graphene heaters photothermally scribed on bare polyimide substrates. Carbon 2019, 144, 116-126.

[77] Huang, X.; Cheng, H. Y.; Chen, K. L.; Zhang, Y. L.; Zhang, Y. H.; Liu, Y. H.; Zhu, C. Q.; Ouyang, S. C.; Kong, G. W.; Yu, C. J. et al. Epidermal impedance sensing sheets for precision hydration assessment and spatial mapping. IEEE Trans. Biomed. Eng. 2013, 60, 2848-2857.

[78] Lan, L. Y.; Le, X. H.; Dong, H. Y.; Xie, J.; Ying, Y. B.; Ping, J. F. One-step and large-scale fabrication of flexible and wearable humidity sensor based on laser-induced graphene for real-time tracking of plant transpiration at bio-interface. Biosens. Bioelectron. 2020, 165 , 112360.

[79] Zhu, C. C.; Tao, L. Q.; Wang, Y.; Zheng, K.; Yu, J. B.; Li, X. D.; Chen, X. P.; Huang, Y. X. Graphene oxide humidity sensor with laser-induced graphene porous electrodes. Sens. Actuators B: Chem. 2020, 325, 128790 .

[80] Adhikari, K. K.; Wang, C.; Qiang, T.; Wu, Q. Polyimide-derived laser-induced porous graphene-incorporated microwave resonator for high-performance humidity sensing. Appl. Phys. Express 2019, 12,106501 .

[81] Xu, Y. D.; Zhao, G. G.; Zhu, L.; Fei, Q. H.; Zhang, Z.; Chen, Z. Y.; An, F. F.; Chen, Y. Y.; Ling, Y.; Guo, P. J. et al. Pencil-paper on-skin electronics. Proc. Natl. Acad. Sci. USA 2020, 117, 18292-18301.

[82] Liu, Y. H.; Pharr, M.; Salvatore, G. A. Lab-on-skin: A review of flexible and stretchable electronics for wearable health monitoring. ACS Nano 2017, 11, 9614-9635.

[83] Xuan, X.; Kim, J. Y.; Hui, X.; Das, P. S.; Yoon, H. S.; Park, J. Y. A highly stretchable and conductive $3 \mathrm{D}$ porous graphene metal nanocomposite based electrochemical-physiological hybrid biosensor. Biosens. Bioelectron. 2018, 120, 160-167.

[84] Choi, S. J.; Kim, I. D. Recent developments in 2D nanomaterials for chemiresistive-type gas sensors. Electron. Mater. Lett. 2018, 14, 221-260.

[85] Wu, D. Z.; Peng, Q. Q.; Wu, S.; Wang, G. S.; Deng, L.; Tai, H. L.; Wang, L. Y.; Yang, Y. J.; Dong, L. X.; Zhao, Y. et al. A simple graphene $\mathrm{NH}_{3}$ gas sensor via laser direct writing. Sensors 2018, 18 , 4405.

[86] Nayak, P.; Kurra, N.; Xia, C.; Alshareef, H. N. Highly efficient laser scribed graphene electrodes for on-chip electrochemical sensing applications. Adv. Electron. Mater. 2016, 2, 1600185.

[87] Xu, G. Y.; Jarjes, Z. A.; Wang, H. W.; Phillips, A. R. J.; Kilmartin, P. A.; Travas-Sejdic, J. Detection of neurotransmitters by threedimensional laser-scribed graphene grass electrodes. ACS Appl. Mater. Interfaces 2018, 10, 42136-42145. 
[88] Han, T.; Nag, A.; Simorangkir, R. B. V. B.; Afsarimanesh, N.; Liu, H. R.; Mukhopadhyay, S. C.; Xu, Y. Z.; Zhadobov, M.; Sauleau, R. Multifunctional flexible sensor based on laser-induced graphene. Sensors 2019, 19, 3477.

[89] Sharma, S.; Ganeshan, S. K.; Pattnaik, P. K.; Kanungo, S.; Chappanda, K. N. Laser induced flexible graphene electrodes for electrochemical sensing of hydrazine. Mater. Lett. 2020, 262, 127150 .

[90] Getachew, B. A.; Bergsman, D. S.; Grossman, J. C. Laser-induced graphene from polyimide and polyethersulfone precursors as a sensing electrode in anodic stripping voltammetry. ACS Appl. Mater. Interfaces 2020, 12, 48511-48517.

[91] Wan, Z. F.; Umer, M.; Lobino, M.; Thiel, D.; Nguyen, N. T.; Trinchi, A.; Shiddiky, M. J. A.; Gao, Y. S.; Li, Q. Laser induced self-N-doped porous graphene as an electrochemical biosensor for femtomolar miRNA detection. Carbon 2020, 163, 385-394.

[92] Yu, Y. C.; Joshi, P. C.; Wu, J.; Hu, A. M. Laser-induced carbon-based smart flexible sensor array for multiflavors detection. ACS Appl. Mater. Interfaces 2018, 10, 34005-34012.

[93] Matsui, J.; Takayose, M.; Akamatsu, K.; Nawafune, H.; Tamaki, K.; Sugimoto, N. Molecularly imprinted nanocomposites for highly sensitive SPR detection of a non-aqueous atrazine sample. Analyst 2009, 134, 80-86.

[94] Jeong, S. Y.; Kim, J. S.; Lee, J. H. Rational design of semiconductorbased chemiresistors and their libraries for next-generation artificial olfaction. Adv. Mater. 2020, 32, 2002075.

[95] Broza, Y. Y.; Zhou, X.; Yuan, M. M.; Qu, D. Y.; Zheng, Y. B.; Vishinkin, R.; Khatib, M.; Wu, W. W.; Haick, H. Disease detection with molecular biomarkers: From chemistry of body fluids to natureinspired chemical sensors. Chem. Rev. 2019, 119, 11761-11817.

[96] Cheng, C.; Wang, S. T.; Wu, J.; Yu, Y. C.; Li, R. Z.; Eda, S.; Chen, J. G.; Feng, G. Y.; Lawrie, B.; Hu, A. M. Bisphenol a sensors on polyimide fabricated by laser direct writing for onsite river water monitoring at attomolar concentration. ACS Appl. Mater. Interfaces 2016, 8, 17784-17792.

[97] Marques, A. C.; Cardoso, A. R.; Martins, R.; Sales, M. G. F.; Fortunato, E. Laser-induced graphene-based platforms for dual biorecognition of molecules. ACS Appl. Nano Mater. 2020, 3, 2795-2803.

[98] Fenzl, C.; Nayak, P.; Hirsch, T.; Wolfbeis, O. S.; Alshareef, H. N.; Baeumner, A. J. Laser-scribed graphene electrodes for aptamer-based biosensing. ACS Sens. 2017, 2, 616-620.

[99] Soares, R. R. A.; Hjort, R. G.; Pola, C. C.; Parate, K.; Reis, E. L.; Soares, N. F.; McLamore, E. S.; Claussen, J. C.; Gomes, C. L. Laser-induced graphene electrochemical immunosensors for rapid and label-free monitoring of Salmonella enterica in chicken broth. ACS Sens. 2020, 5, 1900-1911.

[100] Yoon, H.; Nah, J.; Kim, H.; Ko, S.; Sharifuzzaman, M.; Barman, S. C.; Xuan, X.; Kim, J.; Park, J. Y. A chemically modified laser-induced porous graphene based flexible and ultrasensitive electrochemical biosensor for sweat glucose detection. Sens. Actuators B: Chem. 2020, 311, 127866.

[101] Torrente-Rodríguez, R. M.; Tu, J. B.; Yang, Y. R.; Min, J. H.; Wang, M. Q.; Song, Y.; Yu, Y.; Xu, C. H.; Ye, C.; IsHak, W. W. et al. Investigation of cortisol dynamics in human sweat using a graphene-based wireless mHealth system. Matter 2020, 2, 921-937.

[102] Torrente-Rodríguez, R. M.; Lukas, H.; Tu, J. B.; Min, J. H.; Yang, Y. R.; Xu, C. H.; Rossiter, H. B.; Gao, W. SARS-CoV-2 RapidPlex: A graphene-based multiplexed telemedicine platform for rapid and low-cost COVID-19 diagnosis and monitoring. Matter 2020, 3, 1981-1998.

[103] Stanford, M. G.; Li, J. T.; Chyan, Y.; Wang, Z.; Wang, W.; Tour, J. M. Laser-induced graphene triboelectric nanogenerators. $A C S$ Nano 2019, 13, 7166-7174.

[104] Zhao, P. F.; Bhattacharya, G.; Fishlock, S. J.; Guy, J. G. M.; Kumar, A.; Tsonos, C.; Yu, Z. D.; Raj, S.; McLaughlin, J. A.; Luo, J. K. et al. Replacing the metal electrodes in triboelectric nanogenerators: High-performance laser-induced graphene electrodes. Nano Energy 2020, 75, 104958.

[105] Yang, C.; Huang, Y. X.; Cheng, H. H.; Jiang, L.; Qu, L. T. Rollable, stretchable, and reconfigurable graphene hygroelectric generators. Adv. Mater. 2019, 31, 1805705.
[106] Zhao, F.; Cheng, H. H.; Zhang, Z. P.; Jiang, L.; Qu, L. T. Direct power generation from a graphene oxide film under moisture. $A d v$. Mater. 2015, 27, 4351-4357.

[107] Bai, J. X.; Huang, Y. X.; Cheng, H. H.; Qu, L. T. Moist-electric generation. Nanoscale 2019, 11, 23083-23091.

[108] Zhang, C.; Xie, Y. C.; Deng, H.; Tumlin, T.; Zhang, C.; Su, J. W.; $\mathrm{Yu}$, P.; Lin, J. Monolithic and flexible $\mathrm{ZnS} / \mathrm{SnO}_{2}$ ultraviolet photodetectors with lateral graphene electrodes. Small 2017, 13, 1604197.

[109] Chen, Y.; Long, J. Y.; Zhou, S.; Shi, D. C.; Huang, Y.; Chen, X.; Gao, J.; Zhao, N.; Wong, C. P. UV laser-induced polyimide-tographene conversion: Modeling, fabrication, and application. Small Methods 2019, 3, 1900208.

[110] Hines, L.; Petersen, K.; Lum, G. Z.; Sitti, M. Soft actuators for small-scale robotics. Adv. Mater. 2017, 29, 1603483.

[111] Deng, H.; Zhang, C.; Su, J. W.; Xie, Y. C.; Zhang, C.; Lin, J. Bioinspired multi-responsive soft actuators controlled by laser tailored graphene structures. J. Mater. Chem. B 2018, 6, 5415-5423.

[112] Ling, Y.; Pang, W. B.; Li, X. P.; Goswami, S.; Xu, Z.; Stroman, D.; Liu, Y. C.; Fei, Q. H.; Xu, Y. D.; Zhao, G. G. et al. Laser-induced graphene for electrothermally controlled, mechanically guided, 3D assembly and human-soft actuators interaction. Adv. Mater. 2020, 32, 1908475.

[113] Luong, D. X.; Subramanian, A. K.; Silva, G. A. L.; Yoon, J.; Cofer, S.; Yang, K.; Owuor, P. S.; Wang, T.; Wang, Z.; Lou, J. et al. Laminated object manufacturing of 3D-printed laser-induced graphene foams. Adv. Mater. 2018, 30, 1707416.

[114] Senyuk, B.; Behabtu, N.; Martinez, A.; Lee, T.; Tsentalovich, D. E.; Ceriotti, G.; Tour, J. M.; Pasquali, M.; Smalyukh, I. I. Threedimensional patterning of solid microstructures through laser reduction of colloidal graphene oxide in liquid-crystalline dispersions. Nat. Commun. 2015, 6, 7157.

[115] Kucherenko, I. S.; Sanborn, D.; Chen, B. L.; Garland, N.; Serhan, M.; Forzani, E.; Gomes, C.; Claussen, J. C. Ion-selective sensors based on laser-induced graphene for evaluating human hydration levels using urine samples. Adv. Mater. Technol. 2020, 5, 1901037.

[116] Lin, X. N.; Lu, Z. W.; Dai, W. L.; Liu, B. C.; Zhang, Y. X.; Li, J. Y.; Ye, J. S. Laser engraved nitrogen-doped graphene sensor for the simultaneous determination of $\mathrm{Cd}(\mathrm{II})$ and $\mathrm{Pb}(\mathrm{II}) . J$. Electroanal. Chem. 2018, 828, 41-49.

[117] Li, H.; Guo, C. X.; Liu, C. C.; Ge, L.; Li, F. Laser-induced graphene hybrid photoelectrode for enhanced photoelectrochemical detection of glucose. Analyst 2020, 145, 4041-4049.

[118] Zhang, Y.; Li, N.; Xiang, Y. J.; Wang, D. B.; Zhang, P.; Wang, Y. Y.; Lu, S.; Xu, R. Q.; Zhao, J. A flexible non-enzymatic glucose sensor based on copper nanoparticles anchored on laser-induced graphene. Carbon 2020, 156, 506-513.

[119] Tehrani, F.; Bavarian, B. Facile and scalable disposable sensor based on laser engraved graphene for electrochemical detection of glucose. Sci. Rep. 2016, 6, 27975.

[120] Yoon, H.; Nah, J.; Kim, J.; Xuan, X.; Park, J. Laser-induced graphene stamp for high performacne elecrochemical sensing applications. In Proceedings of the IEEE 32nd International Conference on Micro Electro Mechanical Systems (MEMS), Seoul, Republic of Korea, 2019, pp 537-540.

[121] Zhang, Y. H.; Zhu, H. C.; Sun, P.; Sun, C. K.; Huang, H.; Guan, S.; Liu, H. L.; Zhang, H. Y.; Zhang, C.; Qin, K. R. Laser-induced graphene-based non-enzymatic sensor for detection of hydrogen peroxide. Electroanalysis 2019, 31, 1334-1341.

[122] Aparicio-Martínez, E.; Ibarra, A.; Estrada-Moreno, I. A.; Osuna, V.; Dominguez, R. B. Flexible electrochemical sensor based on laser scribed graphene/Ag nanoparticles for non-enzymatic hydrogen peroxide detection. Sens. Actuators: B Chem. 2019, 301, 127101.

[123] Mamleyev, E. R.; Heissler, S.; Nefedov, A.; Weidler, P. G.; Nordin, N.; Kudryashov, V. V.; Länge, K.; MacKinnon, N.; Sharma, S. Laser-induced hierarchical carbon patterns on polyimide substrates for flexible urea sensors. npj Flex. Electron. 2019, 3, 2.

[124] Yagati, A. K.; Behrent, A.; Beck, S.; Rink, S.; Goepferich, A. M.; Min, J. H.; Lee, M. H.; Baeumner, A. J. Laser-induced graphene interdigitated electrodes for label-free or nanolabel-enhanced highly sensitive capacitive aptamer-based biosensors. Biosens. Bioelectron. 2020, 164, 112272 
[125] Xu, G. Y.; Jarjes, Z. A.; Desprez, V.; Kilmartin, P. A.; TravasSejdic, J. Sensitive, selective, disposable electrochemical dopamine sensor based on PEDOT-modified laser scribed graphene. Biosens. Bioelectron. 2018, 107, 184-191.

[126] Hui, X.; Xuan, X.; Kim, J.; Park, J. Y. A highly flexible and selective dopamine sensor based on Pt-Au nanoparticle-modified laser-induced graphene. Electrochim. Acta 2019, 328, 135066.

[127] Vanegas, D. C.; Patiño, L.; Mendez, C.; de Oliveira, D. A; Torres, A. M.; Gomes, C. L.; McLamore, E. S. Laser scribed graphene biosensor for detection of biogenic amines in food samples using locally sourced materials. Biosensors 2018, 8,42 .
[128] You, Z. H.; Qiu, Q. M.; Chen, H. Y.; Feng, Y. Y.; Wang, X.; Wang, Y. X.; Ying, Y. B. Laser-induced noble metal nanoparticle-graphene composites enabled flexible biosensor for pathogen detection. Biosens. Bioelectron. 2020, 150, 111896.

[129] Cardoso, A. R.; Marques, A. C.; Santos, L.; Carvalho, A. F.; Costa, F. M.; Martins, R.; Sales, M. G. F.; Fortunato, E. Molecularlyimprinted chloramphenicol sensor with laser-induced graphene electrodes. Biosens. Bioelectron. 2019, 124-125, 167-175.

[130] Ge, L.; Hong, Q.; Li, H.; Li, F. A laser-induced $\mathrm{TiO}_{2}$-decorated graphene photoelectrode for sensitive photoelectrochemical biosensing. Chem. Commun. 2019, 55, 4945-4948. 